\title{
Factores de Resiliencia y Afrontamiento como Predictores del Rendimiento Académico de los Estudiantes en Universidades Privadas
}

\author{
Resilience Factors and Coping as Predictors of Academic \\ Performance of the Students in Private Universities
}

\author{
Orlando Tipismana * \\ Universidad Científica del Sur, Perú
}

\begin{abstract}
Predecir el rendimiento académico en estudiantes universitarios peruanos es una garantía para cumplir las exigencias de licenciamiento y acreditación. Se realiza un diseño transversal, comparativo y explicativo para establecer los factores de resiliencia y afrontamiento que predicen el rendimiento académico, en dos muestras probabilísticas estratificadas de universidades privadas de Lima. El RESI-, CAE, Smilkstein, CP-LS y estimadores DWLS, GLS, demostraron que el modelo predictivo de resiliencia y promedio ponderado tienen efecto en la percepción de rendimiento académico y un efecto mínimo en el promedio ponderado. Ambos factores predicen de manera directa, indirecta y relacional el rendimiento académico. Destacan los factores confianza en sí mismo, capacidad social, estrategias de autofocalización emocional abierta, exposición emocional abierta y apoyo familiar social como predictores relevantes en expectativa de logro, aprendizaje percibido y satisfacción global. El funcionamiento familiar, es un predictor indirecto a través de la resiliencia del rendimiento académico. Factores de afrontamiento activo, predicen mejor la percepción del rendimiento que factores de protección y riesgo. Muestras homogéneas grandes con valor psicométrico pueden mejorar los modelos predictivos, identificar perfiles, diseñar estudios causales, desarrollar programas de aprendizaje de talento y estrategias activas que mejorarían la calidad del aprendizaje universitario.
\end{abstract}

Descriptores: Rendimiento escolar; Estudiante universitario; Perú; Calidad de la educación; Enseñanza privada.

Predicting academic performance in Peruvian university students is a guarantee to meet licensing and accreditation requirements. A transversal, comparative and explanatory (predictive) design was used to establish the resilience and coping factors that predict academic performance, in two stratified probabilistic samples from private universities in Lima. The RESI-, CAE, Smilkstein, CP-LS and DWLS estimators, GLS, showed that the predictive model of resilience and weighted average have an effect on the perception of academic performance and a minimal effect on the weighted average. Both factors predict academic performance directly, indirectly and relationally. The self-confidence factor, social capacity, open emotional self-targeting strategies, open emotional exposure and social family support are highlighted as relevant predictors in expectation of achievement, perceived learning and overall satisfaction. Family functioning is an indirect predictor through the resilience of academic performance. Factors of active coping better predict the perception of performance than protection and risk factors. Large homogeneous samples with psychometric value can improve predictive models, identify profiles, design causal studies, develop talent learning programs and active strategies that would improve the quality of university learning.

Keywords: Academic achievement; University students; Peru; Educational quality; Private education.

*Contacto: orlandotipismana@gmail.com

ISSN: $1696-4713$

www.rinace.net/reice/

revistas.uam.es/reice
Recibido: $\quad 15$ de noviembre 2018

$1^{\text {a }}$ Evaluación: 27 de febrero 2019

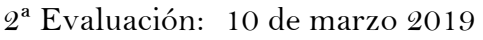

Aceptado: $\quad 25$ de marzo 2019 


\section{Introducción}

Objetivar lo percibido e interpretarlo exige analizar críticamente la realidad académica en pleno siglo XXI (Banco Mundial, 2014). Una sociedad global, la irrupción de las nuevas tecnologías, la introducción de nuevos paradigmas ha permitido comprender las incertidumbres en la educación superior. Estos nuevos retos plantean en el estudiante universitario y sistema educativo el estudio de los modelos mentales que rigen la dinámica del aprendizaje en los contextos universitarios. Haber comprendido que el estudiante es la razón de ser de su aprendizaje se plantea el propósito global de hallar los constructos que mejor expliquen cómo funciona la mente humana en etapa formativa universitaria general, como de las carreras involucradas en ellas.

En este panorama global, la introducción de constructos como resiliencia y afrontamiento académico y su impacto en el rendimiento académico como proceso (percibido) y su resultado (el promedio ponderado) implica necesariamente realizar diagnósticos rigurosos respecto del tipo de perfil personal de los nuevos estudiantes con el objeto de evaluar per se, cómo perciben sus potencialidades, desarrollan o aprenden estrategias y, como impacta en la vida académica, personal social y laboral futura.

De las revisiones efectuadas en el campo educativo, rescatamos dos teorías claves en ese proceso de diagnósticas y evaluar el rol de la resiliencia y el afrontamiento como predictor del rendimiento académico. La teoría sociocognitiva que plantea como fundamento que las personas aprenden observando la realidad a través de cómo los demás aprenden a desarrollar sus potencialidades, crear nuevos recursos, facilitar herramientas para comprender la naturaleza física y social en la que interactúa. Este patrón realidad-mente y viceversa, crea condiciones para modelar los patrones atencionales, motivacionales, de reproducción y de retención de esas realidades, en este caso, se hace referencia a realidades académicas. En otras palabras, los estudiantes desarrollan un sistema de supuestos, opiniones, imaginaciones de cómo es el sistema universitario y el rol que ellos desempeñarían allí. La teoría social cognitiva entonces, se sustenta en el núcleo de creencias y expectativas que es proporcionada por su contexto social, para aprender a interactuar en la vida académica. El estilo de cómo la aprenden y desarrollan sus estrategias, dependerá de tipo de ambiente enriquecido o no para estructurar un perfil personal que los apoye en ese proceso de desempeño académico en la universidad (Tadayon, 2012).

En cambio, la teoría de la autoeficacia se fundamenta en los juicios que se van creando a partir de esa realidad académica que aprenden antes de ingresar a la universidad y de la manera cómo incorporan las experiencias de la universidad a ese sistema de creencias y expectativas creadas. Es decir, los estudiantes aprenden a tener control de sí mismo, desarrollar un patrón social, estructurar una personalidad y desarrollar una estructura de pensamiento que orientará toda su vida a la búsqueda de logros de metas. Dependiendo de las habilidades y destrezas que desarrolle en su sistema cognitivo (pensamientos, atención, memoria, reproducción), implantará un sistema de creencias y expectativas basadas en juicios de valor, motivaciones, actitudes, valoraciones, control emocional, patrones evitativos, espiritualidad entre otros. A estos patrones o desarrollos de metas y logros, se estructurará un patrón mental eficaz o no.

La teoría social cognitiva del aprendizaje vendría a ser el punto de partida de aprendizajes sociales modelados y moldeados por la cultura familiar, social, escolar y universitaria para 
ser autoeficaz o no. En ese marco de reflexión es que los patrones cognitivos se convierten en la estructura visible del pensamiento del estudiante y, los patrones no cognitivos (creencias y expectativas) en la estructura no visible y necesaria de diagnosticar y evaluar para poder perfilar el tipo de estudiante que la universidad tendrá en el futuro próximo.

La universidad, al fomentar la búsqueda de perfiles de estudiantes con potencialidades y estrategias para interactuar en un contexto académico de alta complejidad, requiere identificarlas y diseñar estrategias que al brindar sus servicios puedan garantizar la formación del estudiante, conociendo previamente ese patrón de creencias y expectativas para transformarlas o, moldearlas con el objeto de obtener recursos humanos altamente competentes. Y que, los servicios que brinden sean sostenibles en el tiempo, no solo durante la vida académica, sino que, trascienda a la vida laboral (Comisión Europea, 2015; OCDE, 2013; Sucre y Garre, 2016).

En consecuencia, estos rasgos que definen las creencias y expectativas pueden ser explicadas por los constructos de resiliencia y afrontamiento académicos. Ello demanda predecir de manera objetiva y confiable si los rasgos identificados en el perfil del estudiante pueden asegurar que su aprendizaje estará orientado a adecuarse, adaptarse y lograr las metas esperadas por él como por la universidad.

Los modelos predictivos entonces, trazan una nueva línea de investigación en la universidad. Estos puedan diseñar y desarrollar programas académicos que identifiquen y valoren los atributos que tiene un estudiante para que sus creencias y expectativas de logro sean satisfechas (Cabanach et al., 2010; Limonero et al., 2012; Piemontesi y Heredia, 2009).

\section{Revisión de la literatura}

Desde este marco explicativo de la manera como los estudiantes aprenden de su entorno, desarrollan creencias y expectativas de logro, es importante analizar los tres constructos para identificar esos factores inherentes y que impactan en la vida académica universitaria.

\subsection{Resiliencia académica}

Se entiende por resiliencia académica al desarrollo de recursos potenciales que emplea el estudiante universitario para resolver problemas y tomar decisiones en situaciones típicas de la vida universitaria (Yan Lee, Cheung y Man Kwong, 2012). También este aprendizaje implica procesos y resultados. Que se expresen de manera eficiente tanto en sus aspectos cognitivos como no cognitivos.

La resiliencia, por consiguiente, perfila el alcance de metas y logros, plantea exigencias para la construcción de nuevos recursos, permite al estudiante utilizarlos con regularidad en diversas situaciones y contextos donde interactúe. Esta interacción será eficaz, en la medida que sus expectativas de logro sean consistentes, congruentes y pertinentes. En otras palabras, aprender a percibir la tenencia de recursos impulsará su aprendizaje y facilitará del manejo de las incertidumbres y la búsqueda de certezas para tener el control de la situación. El propósito es, poder autorregularse de manera continua a través del tiempo, así como sostenible cognitiva y emocionalmente (Becoña, 2006; Datu y Yuen, 2018).

Una manera de corroborar este presupuesto de creencias y expectativas de logro para percibir si se es o no eficaz, tiene que ver con el uso de las nuevas herramientas 
tecnológicas, en este nuevo escenario académico se introducen metodologías como la flipped classroom; el problema no está en la metodología misma. La dificultad estaría en el estudiante. Es decir, cómo percibe el uso de esta herramienta de aprendizaje, por qué es importante la inversión de roles, que logros se pretende obtener, no solo es una cuestión de utilidad, sino de la percepción de aprendizaje y la expectativa de logro además de si genera o no satisfacción en él (Sola Martínez, Aznar Díaz, Romero Rodríguez, RodríguezGarcía, 2019). La resiliencia como recurso potencial, entonces, parte de la idea que calidad del logro es más que un asunto personal, implica lo que se denomina "un aprendizaje personalizado” (Farnós Miró, 2013). Este aprendizaje personalizado, dependerá de la capacidad de los actores para consensuar los intereses, motivaciones, refuerzos, respuestas emocionales, respuestas adaptativas a cualquier tipo de cambio y, a su manera de percibir y controlar los eventos o acontecimientos estresantes.

Un estilo de respuesta resiliente es esencial para identificar en el estudiante el ¿ipor qué? Porque las experiencias de dominios mejoran los estados afectivos, generan nuevas necesidades de aprendizajes, pero, sobre todo, de nuevas creencias de logros, mejoras en sus percepciones y poder afrontar de manera flexible el entorno y la situación (Bandura, 1989). Lo que se busca es, estar en sintonía entre esas creencias y expectativas de logro y las actividades de la vida académica. Que no solo estimule nuevas necesidades de aprendizajes, también, de mayor confianza y control de respuestas de humor.

Entonces, la predictibilidad de un patrón personalizado en un estudiante resiliente implica que en el presente y sus proyecciones hacia el corto o mediano plano, se implante un estilo de persistencia que impacte en la búsqueda de nuevos recursos para aprenderlos, tener satisfacción, incrementar sus creencias racionales, extender su aprendizaje a la familia y a su entorno social próximo (Lamas, 2015; Medrano, 2011; Porto Noronha y Alves Lamas, 2014). En este sentido, la extensión de su aprendizaje involucra el desarrollo de talentos para la relación interpersonal ya sea con sus compañeros, mejorar la pasión por el trabajo académico, la alegría y amor por lo que hacen (Crowther y Lau, 2019).

Todos estos factores no cognitivos (persistencia, pasión por el trabajo, amor por lo que hace, expectativa de autoeficacia, control, autorregulación cognitiva y emocional), involucran cambios en la percepción de controlabilidad de la situación y de sí mismo, de ajuste a la situación y de adecuación a las demandas del entorno (Gan y Shang, 2007). Por esta razón, es que los factores cognitivos y no cognitivos, interactúan de manera dinámica y sintonizada. Ayudando a generar patrones de protección y de adecuada visualización efectiva de sus acciones, para evitar riesgos o asumir riesgos, sostenidos por recursos de control emocional que garanticen el éxito académico (Prieto Navarro, 2006).

Diferentes autores han desarrollado estudios predictivos, reportan que la mejora de atributos personales, desarrollo de nuevos recursos, mejora en el nivel de resistencia al estrés, control de la ansiedad y angustia se explica por los altos niveles de atención y, su repercusión será significativa en los logros personales que obtengan en la vida académica y social. Estos rasgos, predicen mejor el rendimiento académico (López-Cortón, 2015). También los factores cognitivos y metacognitivos se asocian a la llamada "inteligencia emocional", a las motivaciones. Pero, sobre todo, en la definición de objetivos de logro que va implantándose a medida que aprende nuevos recursos y garantice su aprendizaje de una materia, de una técnica académica, mejor relación con sus pares y garantice su eficacia cognitiva, emocional y conductual (Giannuzzo, 2010; Kormos, 2012). 
Otros autores sostienen que la mejora en las potencialidades implica un mejor involucramiento con la familia, adecuación a las normas académicas y sociales incrementan el compromiso personal (Weier y Lee, 2015). También en la mejora del estudiante en su relación con el docente durante la actividad académica (Uriarte, 2006), potencian las habilidades cognitivas (Girardo et al., 2016).

Finalmente, otros estudios predictivos demuestran, que las mejoras en la capacidad de autorrecompensa incrementan la habilidad para participar activamente en la vida académica cotidiana, desarrolla compromisos consigo mismo, y contribuye a estructurar una mejor competencia social (Garbanzo Vargas, 2013; Mancini y Bonanno, 2009). La motivación, articula mejor los estados de ánimo y las actividades académicas, favorece un mejor clima de relación entre todos los actores académicos. Estos, se estructuran eficientemente en un entorno saludable, repercutiendo de manera valiosa en el proceso de aprendizaje y enseñanza e incluso, a predecir su futuro laboral (Kennett y Keefer, 2006).

\subsection{Afrontamiento académico}

Vendrían a ser estilos o maneras como un estudiante al tener recursos potenciales, desarrolla habilidades para ajustarse a una situación determinada. Es decir, si el estudiante aprende que las situaciones cambiantes de un ambiente académico demanda focalizarse en el problema, es evidente que esa focalización, implicara un mejor valor académico para alcanzar sus metas propuestas. El afrontamiento, no solo es adaptación, implica el desarrollo de estilos cognitivos y conductuales. Los hallazgos indican que la capacidad de percibir la situación, evaluar su desempeño, desarrollar actividades de emprendimiento para planificar mejor su tiempo, usar recursos académicos, desarrollar nuevos esquemas mentales de trabajo, tienen un objetivo común, alcanzar metas propuestas (Muñoz Garrido y De Pedro Sotelo, 2004).

Los estilos de afrontamiento son en sí formas de actuar en situaciones y contextos cambiantes y difíciles como suele ser la vida académica universitaria. Este patrón de acciones y pensamientos tienen un carácter multidimensional al igual que la resiliencia. Algunos autores lo denominan "afrontamiento resiliente" o, también, "afrontamiento flexible”. Esta manera de enfocar el papel del afrontamiento indica que su relación con la resiliencia puede ser de acción directa a la vida académica o, frecuentemente va asociada a la resiliencia para incidir de manera eficaz en el rendimiento académico (Martin y Marsh, 2009). También, se asocia con estilos activos o estilos pasivos. Un estilo activo se relaciona más con la reevaluación positiva de sus acciones, la focalización centrada en la solución de problemas y una reevaluación positiva. En cambio, los estilos pasivos, se orientan más a rasgos de actuación orientadas a buscar apoyo social, la espiritualidad, autofocalización negativa (Rebotier, López y Pigeon, 2013)

En consecuencia, el afrontamiento entendido como toda capacidad de determinación de un estudiante que lo impulsa a, asegurar con su estilo (por ejemplo: activo), el dominio de la situación estresantes en el contexto académico, implica adaptación a la situación que asegure su expectativa de logro, alcanzar metas y ayudar a gestionar convenientemente su persona en toda actividad académica. Un estudiante con un afrontamiento resiliente no ve su vida limitada a la vida universitaria, sino que su quehacer académico va más allá de ella misma. Un estilo de afrontamiento activo orientado a la solución de problemas predice mejor el rendimiento académico a partir, del mayor compromiso que asume para lograr la solución específica a un problema planteado (Coschiza et al., 2016). 
El compromiso, está relacionado más con la confianza en sí mismo, ayuda a desarrollar estrategias adaptativas para manejar respuestas emocionales negativas, involucrar respuestas positivas y mejorar todas las actividades que aseguren el éxito académico. No obstante, hay afrontamientos negativos como le autofocalización negativa y estilo evitativo que, si no son adecuadamente gestionados por el estudiante, pueden generar mayores respuestas fisiológicas negativas, pérdida de la homeostasis e interferencia en la capacidad percibida de ajuste ante situaciones conflictivas. Además, de deterioro en la capacidad física y mental (Campos et al., 2004; Rahimi et al., 2014).

Por otro lado, diversas observaciones en el entorno académico, se ha encontrado que un estudiante cuando aprende a gestionar el estilo de autofocalización negativa y adopta una mejor capacidad social (factor resiliente), este patrón predice un mejor rendimiento académico. Sobre todo, cuando se aprende a tener control emocional, adaptación a diversas situaciones, que ayudará a mejorar su desempeño académico y personal (Knezevíc y Knezevíc, 2016; Panziera, 2014).

En consecuencia, el afrontamiento académico se comporta más como un predictor mediador o indirecto del rendimiento académico. Los estilos de afrontamiento activos suelen ser más adaptativos que los afrontamientos pasivos, aun cuando, en estudios recientes, se toma en cuenta mucho el factor espiritualidad, estilo que mejora o ayuda positivamente a muchos estudiantes a autorreforzarse para obtener logros. Sobre todo, cuando no ha asumido el valor de su potencialidad o, sus aprendizajes en el uso de esos recursos requieren de más estimulación externa y, eso tendría mucho que ver con el locus de control externo. Es decir, muchos estudiantes necesitan ser motivados emocionalmente para poder producir académicamente. También se asocia con lo que se conoce recientemente sobre flotabilidad académica (bouyance academic), es decir, los estudiantes a pesar de la adversidad o aparente carencia de recursos personales aprenden a salir a flote y autorregularse. El asunto es, replantear sus planes personales en el desempeño académico (Tomás et al., 2012).

\subsection{Rendimiento académico}

Suele definirse regularmente como obtención de resultados a partir de la actividad didáctica que emplea un docente. Sin embargo, en años recientes, el rendimiento académico implica, el logro de un complejo proceso de aprendizajes en expectativas de éxito, mejor visualización en la percepción de su aprendizaje y la satisfacción de haber obtenido resultados eficientes. Estos, motivan a desarrollar nuevas o las mismas actividades, para adquirir un rol destacado y recompensar su desempeño (Lamas, 2015).

Es decir, el rendimiento académico, es un conjunto de procesos no cognitivos que articulados a los cognitivos predicen mejor el logro de metas y objetivos académicos planeados por el estudiante o, por la demanda del entorno académico. En el desempeño académico, se articulan en especial las creencias, expectativas, percepciones y potencialidades. Indicadores que expresan de manera real, las estrategias adaptativas y estilos de afrontar diversas situaciones (Guzmán Brito, 2012).

Estas percepciones cuando se relacionan con el rendimiento académico implican el resultado de una estructuración personal, caracterizada por el desarrollo de diversas actividades académicas que no solo se orientan al plano didáctico, también a enriquecer su relación con el docente, compañeros de clase y miembros de la comunidad universitaria. El rendimiento académico por sí mismo, no se puede relacionar directamente a 
evaluaciones, calificaciones. Este constructo, se debe asociar principalmente con el desarrollo de dominios, mejoras en la percepción y expectativa de logro. Garantía de que la percepción de logro conducirá o llevará al estudiante a ser autoeficaz cognitiva y activamente en la demanda natural de las actividades académicas en función de los planes curriculares como a la adaptación de normas y reglamentos. Lo central está en la garantía percibida de autoeficacia, autorregulación y ajuste emocional, que da sostenibilidad a la integración de sus potencialidades y estilos estratégicos en su actuar (Kötter et al., 2014).

Muchos autores, relacionan al rendimiento con el logro de gestión de emociones, ajuste en las cogniciones y dominios de comportamientos no solo académicos, también, con la capacidad para percibir la realidad, entender la incertidumbre, mejorar la atención, obtener logros cognitivos, metacognitivos y mejoras sostenibles de pensamiento y aprendizajes (Cheng, Ickes y Verhofstadt, 2012). Por lo tanto, no se debe descuidar que la relación entre potencialidad, estilo comportamental y cognitivos implica, que el rendimiento académico es más un proceso activo y proactivo de ayuda a los aprendizajes. Que se expresen con mayor seguridad y mejor desempeño con continuidad, transversales y escalables a mayores niveles de complejidad. La incorporación de estos factores no cognitivos al rendimiento académico, implican que el proceso de cambios en la estructura sociocognitiva y de autoeficacia como lo postuló Bandura, comprometen más a los actores para desarrollar esfuerzos multidisciplinarios orientados a consolidar el conocimiento y la práctica activa en productos realmente necesarios para la sociedad en su conjunto. Se busca estructurar mejor la autonomía del estudiante, contrarrestar el fracaso académico (Peralta Díaz, Ramírez Giraldo y Castaño Buitrago, 2006), y regular la percepción de que las calificaciones son una consecuencia derivada de estos procesos no cognitivos y cognitivos (Rodríguez et al., 2011).

\subsection{Factor familiar}

La familia, es un vehículo notable para la mejora en los aprendizajes de recursos personales y de estilos de afrontamiento, motivados por el patrón de moldeamiento y modelamiento de los estudiantes a lo largo de la vida previa al sistema académico universitario. Un patrón disfuncional, dificultará su desempeño, aunque no necesariamente obstaculizará el desarrollo de nuevas estrategias de aprendizaje. Siempre habrá posibilidades nuevas para manejar y ajustarse adecuadamente a las incertidumbres, las adversidades y la complejidad de la vida académica.

Un estudiante con un patrón familiar funcional estará en mejores condiciones de asegurar sus recursos y afrontar la vida académica, en condiciones favorables que los estudiantes con disfuncionalidad familiar (Rivas et al., 2017). El factor familiar, es un factor decisivo en la resiliencia y el afrontamiento (Muñoz Garrido y De Pedro Sotelo, 2004). El factor familiar, se construye como un patrón ecológico externo al sistema universitario y su repercusión será un predictor clave del papel de los padres, amigos y del aseguramiento de la vida académica universitaria, así como de hacerlo sostenible (Campo Vásquez et al., 2012).

El factor familiar, asociado a los constructos de resiliencia y afrontamiento será entonces, un predictor válido en la atención, pensamiento, procesos sensoriales, toma de consciencia, aceptación, desarrollo de fortalezas, identificación de debilidades. El nivel de ajuste y la regulación del estudiante para logros académicos eficaces serán decisivas en el proyecto personal presente y futuro (López-Vargas, Hederich-Martínez y Camargo-Uribe, 2011). 
En cuanto a los estilos de relaciones familiares, un patrón relacional ayuda significativamente en el ajuste personal social y en la toda acción académica (Campos et al., 2004; Rahimi et al,. 2014).

La resiliencia y el afrontamiento, como factores inherentes al estudiante y su correlato con la percepción de relación familiar, definirá cómo será el patrón cognitivo y probablemente influirá en la capacidad de trascender su vida formativa, en la ayuda a los tipos de aprendizaje (Cassaretto y Martínez, 2009). La toma decisiones, por ejemplo, es un estilo no cognitivo que, aprendida principalmente en el entorno familiar, definirá la toma de decisiones ajustadas a la realidad académica, personal y social; incrementará el factor optimismo, la perseverancia, además del interés por el optimismo y las relaciones positivas con sus pares y docentes. Estos factores, crean un sistema de pensamiento creativo, capaz de superar adversidades propias de la vida académica, familiar y social e interactuar con la diversidad (Kuo, 2014); permitirá adaptarse a nuevas realidades académicas e incorporar componentes claves para la solución de problemas (Stocker y Faría, 2012).

Por último, predecir la influencia del factor familiar en la resiliencia y el afrontamiento en el RA, implicará que la universidad, deberá evaluar la estructura y dinámica familiar, no solo en el proceso de admisión, también en toda la vida académica, identificando problemas, detectando como afecta su perfil personal y cómo impacta en los aprendizajes (Martin y Marsh, 2009). Permitirá que el sistema universitario, desarrolle planes más flexibles que ayuden al aprendizaje global de los estudiantes; mejorar la oferta y política educativa, como en los procesos académicos y complementarios (Luthar, Cicchetti y Becker, 2000).

\section{Método}

El objetivo central de esta investigación es establecer si los factores de resiliencia y afrontamiento predicen el rendimiento académico en estudiantes universitarios

Objetivos específicos

- Identificar si los factores psicosociales (funcionamiento familiar, factores de protección, riesgo y, afrontamiento pasivo y activo), predicen el rendimiento académico

- Identificar el factor sexo y especialidad académica, predicen la percepción de rendimiento académico

Hipótesis de trabajo

- Los factores de resiliencia y afrontamiento predicen positiva y significativamente el rendimiento académico en estudiantes universitarios

- Los factores psicosociales como el factor familiar, el factor de protección y afrontamiento activo, predicen el rendimiento académico en estudiantes universitarios.

- El sexo y la especialidad académica impactan positiva y significativamente en el rendimiento académico en estudiantes universitarios 


\section{Enfoque metodológico}

Esta investigación cuantitativa, de corte transversal, comparativo y explicativo en su análisis, buscó establecer si los constructos de resiliencia y afrontamiento predicen el rendimiento académico en estudiantes de ciencias de la salud comparado con estudiantes de ciencias empresariales de dos universidades privadas de Lima, Perú, en el periodo lectivo 2017-I. Es pertinente precisar, que, al ser un estudio transversal, las predicciones de los constructos se orientaron a desarrollar modelos teóricos de tipo estadístico, basado en la teoría sociocognitiva. Para establecer este patrón predictivo, se buscó identificar la influencia directa, indirecta y correlacional de los factores de resiliencia, afrontamiento y el rendimiento académico (Bernal, 2012; Escobedo et al., 2016).

\section{Variables}

Las variables explicativas, son los factores de resiliencia y afrontamiento para establecer si predice el rendimiento académico consideradas como variable criterio (percepción de rendimiento) y el promedio ponderado. En variables de control, se buscó identificar factores psicosociales como: funcionamiento familiar; factores protectores y de riesgo; factores de afrontamiento activo y pasivo. En variables sociodemográficas: sexo y especialidad académica.

\section{Muestra y muestreo}

Estudiantes de Ciencias de Salud (Medicina, Enfermería y Tecnología) $[\mathrm{n}=465]$ y Ciencias Empresariales (Ingeniería de Sistemas Empresariales, Ingeniería Económica, Administración de Negocios Internacionales) $[\mathrm{n}=131]$ de dos universidades privadas de Lima Metropolitana. Las muestras, se obtuvieron mediante muestreo probabilístico estratificado; nivel de confianza para CC.SS de $5 \%$ y de $7 \%$ para CC.EE. Para los criterios de selección, se tuvo en cuenta solo a estudiantes matriculados regulares (mayor de 18 créditos) en el semestre académico 2017-I, que conserven la misma condición durante el semestre académico 2017-II, de los ciclos lectivos II al X, para poder obtener el promedio ponderado semestral. Las edades, se distribuyeron en dos grupos de 16 a 21 años y de 2227 años, sexo masculino y femenino.

\section{Instrumentos de obtención de información}

Escala de Resiliencia Académica: (RESI-M), con 43 ítems, distribuidos en cinco dimensiones. Dimensión 1: Fortaleza y Confianza en Sí mismo 18 ítems; Dimensión 2: Competencia Social, 8 ítems; Dimensión 3: Apoyo Familiar, 5 ítems; Apoyo Social: 4 ítems; Dimensión 5: Estructura Personal, 5 ítems. El autor toma como referencia el CD-RISC de Connor y Davidson (1999); RSA de Friborg y otros (2001), citado por Palomar y GómezValdez (2010). Cuenta con cinco opciones de respuesta en escala Likert de 1 a 4 . Se modificó a una escala de 1 a 5.1 (nunca) hasta 5 (casi siempre). Un índice de validez de contenido aceptables 0,79. La varianza extraída total es de $58,71 \%$.

Cuestionario de Afrontamiento al Estrés (CAE). Sandín y Chorot (2003, construyen el instrumento con 42 ítems; una escala Likert de 1 a 5, distribuidas en 7 factores con seis ítems cada uno. Factor 1: Focalizado en la solución del problema (FSP) 6; Factor 2: Autofocalización negativa (AFN); Factor 3: Reevaluación Positiva (REP); Factor 4: Exposición emocional abierta (EEA); Factor 5: Estilo de evitación (EVIT); Factor 6: Búsqueda de Apoyo Social (BAS); Factor 7 Espiritualidad (ESP). Los coeficientes Alfa de 
0,77 a 0,84 en los 7 factores; el índice de validez de contenido aceptables 0,80. La varianza total extraída fue $58,3 \%$

Escala de Funcionamiento Familiar de Smilkstein, es una escala con una sola dimensión abreviada de 5 ítems, con valoraciones en una escala Lickert de 1 a 5 (adaptada de Castilla et al., 2014). El coeficiente alfa de Cronbach presenta valores alfa 0,80. El índice de validez de contenidos aceptables, $56,4 \%$; y la varianza total extraída de $56,4 \%$.

Escala de Rendimiento Académico. Se integró dos instrumentos distribuidas en 3 factores con 28 ítems en total, utilizando una escala Likert de 1 a 4 puntos. La Dimensión 1: Expectativa de Logro (8 ítems); Dimensión 2: Aprendizaje Percibido (10 ítems); Dimensión 3: Satisfacción global 8 ítems. La Confiabilidad global 0,86 y para los coeficientes alfa oscila entre 0,81 a 0,86 para cada dimensión. El índice de contenido aceptables $59,1 \%$; una varianza total extraída de $59,1 \%$.

La adaptación de todos los instrumentos se realizó, con una prueba piloto de 190 estudiantes en Ciencias de la Salud y Ciencias Empresariales de dos universidades privadas de Lima, Perú. Se siguió un procedimiento de aplicación mix to (heteroaplicada y auto aplicada). Los estudiantes, accedieron voluntariamente responder al cuadernillo utilizado, previa a la presentación de la carta de consentimiento informado.

En importante precisar, que las escalas de Likert pueden variar en su carácter escalar. Si bien, la mayoría de los autores utilizan escalas ordinales de 1 a 5 o más; otros autores, consideran valores de 0 a $4 / 1$ a 4, lo que no compromete la equidistancia en el valor ordinal de los datos (Palomar y Gómez Valdez, 2010; Rodríguez, Oñate y Mesurado, 2017; Sandín y Chorot, 2003). De igual manera, los autores originales de los instrumentos adaptados utilizaron varianza acumulada extraída con valores obtenidos parecidos a este estudio. No obstante, es conveniente especificar que las diferencias entre los estudios para adaptación de instrumentos; pueden diferir de estudios que requieren validez psicométrica. Claro está, que debe cumplirse con el parámetro pertinente para estudios en psicología, educación y ciencias sociales. Estas varianzas, debe ser mayor al $65 \%$. En este trabajo de investigación, se buscó adaptar ambos instrumentos y en base a esos valores obtenidos, desarrollar a futuro estudios de validez psicométrica de los tres instrumentos relacionados con: Rendimiento Académico, Resi-M y CAE.

\section{Trabajo de campo}

Se siguieron los siguientes pasos:

Paso 1. A través del director de la Escuela de Ingeniería de Sistemas empresariales de la Facultad de CC.EE, se solicitó aula por aula y mediante consentimiento informado que los estudiantes, responden al cuadernillo de cuestionarios seleccionados. El proceso duró 3 días para la prueba piloto y 15 días para recolectar la información con la ayuda de dos estudiantes de la Asignatura de Seminario de Tesis y Gestión por Competencias. De las 5 escuelas, se seleccionaron 3 para conciliar los criterios de ajuste de selección de estudiantes. Se eligieron a estudiantes del II a X. Estos fueron: Ingeniería de Sistemas Empresariales, Ingeniería Económica y Administración de Negocios Internacionales.

Paso 2. En la Facultad de CC.SS de otra universidad privada, se contó con el apoyo de la directora de la Escuela de Ciencias Médicas y Fisioterapia, quien ayudó de intermediaria para acceder a todos los estudiantes de: Medicina, Enfermería y Tecnología Médica. 
Paso 3. Se revisaron los instrumentos para verificar la idoneidad del llenado de los ítems y datos generales. De 147 estudiantes de una población de 630 estudiantes con un nivel de confianza de p. $5 \%$, fue reajustada a $7 \%$ por la eliminación de 16 estudiantes; los datos eran incompletos en tres escuelas de CC.EE. En CC.SS, se obtuvo una muestra de 465 estudiantes mediante el muestreo probabilístico estratificado, de una población de 1,807 estudiantes; el nivel de confianza para esta muestra es de p. $5 \%$

Paso 4. Se eligieron 12 jueces entre docentes de medicina 5; psicología 4; y de ingeniería 3. A cada juez, se remitió el certificado de validación de jueces. Datos que se presentan en la sección de resultados en la modalidad de CVI global y aceptables y, varianza extraída.

Paso 5. Posteriormente, se elaboraron la base de datos en una hoja Excel, para identificar las puntuaciones y codificar las dimensiones de cada instrumento.

Paso 6. Se trabajó con el software libre R con la librería lavan. En este paquete estadístico se utilizaron estimadores específicos, para análisis SEM. Los modelos de ecuaciones estructurales se construyeron el semPlot del R comander.

\section{Análisis de datos}

Las pruebas de normalidad para cada instrumento. Los valores $0,010<0,05$, indican que ambas muestras son poblaciones no normales. La prueba de Kolmogorov-Smirnov (K-S) fue el estadístico de elección.

Los datos descriptivos, fueron planteados bajo el supuesto de puntos de corte automático mediante el método de datos categorizados en SPSS. Además de frecuencias y porcentajes, se realizaron análisis descriptivo, medidas de tendencia central, desviación estándar y prueba de significancia de U Mann-Whitney.

El tratamiento de los datos para los modelos SEM se realizó con el Método de Mínimos cuadrados ponderados diagonales mix tos (PLS) y los estimadores DWLS y GLS.

Para la fiabilidad de los instrumentos, se utilizó el Coeficiente alfa de Cronbach. Para validez de contenido por criterio de jueces, se usó la Validez de Contenido Global y específico de Lawshe. Para obtener el AFE, fue conveniente utilizar la prueba KMO; la prueba de ji cuadrado, la prueba de esfericidad de Bartlett y la varianza acumulada según el método de Moriyama. Procedimientos combinados, para garantizar la adaptación confiable y robusta de los cuestionarios y poder aplicarse en los estudiantes de carreras elegidas.

El AFC, se utilizaron los indicadores de bondad de ajuste: Absoluto, incremental y de parsimonia. Además, las matrices de variables latentes, covarianzas y varianzas; análisis de regresión lineal para obtener los $\beta$ y la t y nivel de significación (p-valor). Sin embargo, era importante, realizar una post prueba confirmatoria para disminuir sesgos estadísticos. Por tal razón, se utilizaron la prueba de fiabilidad compuesta y la varianza extraída promedio para obtener la validez convergente.

El Contraste de hipótesis de los objetivos generales y específicos, se eligió la matriz de regresiones lineales y el contraste de hipótesis para evaluar los $\beta$ estandarizados y, el valor de t, y significancia estadística (p-valor $0.000 \leq 0,05$ ). La prueba de Bootstrapping para el análisis de mediación directa, indirecta, de efecto y proporcional (Antal y Tillé, 2011). La invarianza métrica, fue usada para establecer la percepción respecto de sexo y especialidad académica. Por último, se presentan los diagramas de senderos (SemPlot) modificados para objetivos generales y específicos y tablas de bondad de ajuste y contraste de hipótesis. 


\section{Resultados}

Según los objetivos e hipótesis planteadas, se presentan los resultados del análisis de confiabilidad y validez de los instrumentos para adaptarlos al contexto de estudio (AFE, AFC, Bondad de Ajuste, Análisis de variables latentes, covarianzas, y un análisis post confirmatorio para estabilizar la adaptación de los instrumentos usados en este caso). Se describen los datos, con puntajes por punto de corte automático categorizados. El desarrollo del modelo teórico es descrito, y se presentan los datos de análisis de: predicciones de los factores resiliencia y afrontamiento en la percepción de rendimiento académico y promedio ponderado; la prueba de sensibilidad del efecto directo, indirecto y mediacional (Bootstrapping). Se analizan el papel de los factores psicosociales (funcionamiento familiar, factores protectores de protección, riesgo; factores de afrontamiento activo y pasivo). Así como el rol de los factores sociodemográficos como sexo y especialidad académica del estudiante para valorar la percepción respecto de los constructos y su impacto en el rendimiento académico.

\subsection{Análisis de confiabilidad y validez de los instrumentos}

\section{Análisis factorial exploratorio}

La medida de adecuación al muestreo (KMO) presenta valores de 0,80 hasta 0,94 [IC 95\% 0,80-0,99] (cuadro 1) y, la prueba de esfericidad de Barlett, confirma la adaptación de los instrumentos utilizados. Este valor significativo, corrobora la aplicabilidad de los cuestionarios a ambas muestras y que, provienen de la población de estudiantes en CC.Ss y CC.EE $(0,000 \leq 0,05)$. Al cumplir el propósito de adaptación de los instrumentos, se hizo posible realizar, la prueba $\mathrm{AFE}$.

Cuadro 1. Prueba de KMO y Bartlett

\begin{tabular}{lcrcc}
\hline & \multirow{2}{*}{ KMO } & \multicolumn{3}{c}{ PRUEBA DE BARTLETT } \\
\cline { 3 - 5 } & & \multicolumn{1}{c}{$\mathbf{X}^{2}$} & gl & Sig. \\
\hline Resiliencia & 0,94 & 6526,163 & 903 & $0,000 \leq 0,05$ \\
Afrontamiento & 0,85 & 2722,553 & 861 & $0,000 \leq 0,05$ \\
Funcionamiento familiar & 0,80 & 632,020 & 10 & $0,000 \leq 0,05$ \\
Rendimiento académico & 0,92 & 2469,347 & 378 & $0,000 \leq 0,05$ \\
\hline
\end{tabular}

Nota: KMO Prueba de Kaplan Meyer y Olkin; X² Chi cuadrado; gl Grados de Libertad; Sig. Nivel de significación.

Fuente: Elaboración propia.

$\mathrm{El}$ análisis factorial exploratorio (AFE) (cuadro 2). Los coeficientes alfas en la prueba piloto $(\mathrm{n}=190$ estudiantes de CC.SS (60,5\%) y CC.EE (44,7\%); por dimensiones oscilan de 0,72 a 0,91 [IC\% 0,70-0,95]; los coeficientes alfas globales de 0,77 a 0,87 [IC 95\% 0,75-0,90]; y, con el factor rotado Varimax promediados de 0,75 a 0,87 [IC 95\% 0,70-0,90], demuestran la pertinencia de los instrumentos. Es decir, se toman en cuenta el número de ítems; garantizan la pertinencia de un intervalo mínimo apropiado, apoyado por la prueba KMO; se asegura una muestra estratificada conveniente; cumplen con la prueba de esfericidad y, un p-valor altamente significativo (Frías-Navarro y Soler, 2012). El juicio de expertos demuestra por otro lado, que los coeficientes de validez de contenido global y aceptables de Lawshe (1975) modificado por Tristán (2007, citado en Tristán-López y MolgadoRamos, 2007) son aceptables. Valores de 0,71 a 0,80 [IC 95\% 0,67-0,85], aseguran la validez de contenido. Los valores del test de Moriyama en relación con la varianza 
acumulada va de $56,4 \%$ a $62,6 \%$; éstos son datos cercanos a los valores de los autores originales de cada instrumento (Castilla, Caycho, Shimabukuro y Valdivia, 2014; Gargallo López et al., 2009; Olivas y Barraza, 2016; Sandín y Chorot 2003). Quizás, esta diferencia menor a $65 \%$ permitido para ciencias sociales, puede afectar la robustez estadística; sin embargo, se encuentra en diferentes estudios (como los citados), que es común el uso de estos valores. Es necesario precisar, que, en este caso, no se realiza un estudio de análisis psicométrico; solo se están adaptando los instrumentos originales.

Cuadro 2. Coeficientes alfa; factores rotados promedio; CVI y varianza acumulada

\begin{tabular}{|c|c|c|c|c|c|c|c|}
\hline & $\mathbf{F}$ & $\mathrm{CA}$ & CAG & $\begin{array}{c}\text { F. } \\
\text { RVXP. }\end{array}$ & $\begin{array}{c}\text { CVI } \\
\text { GLOBAL }\end{array}$ & $\begin{array}{c}\text { CVI } * \\
\text { ACEPTABLES }\end{array}$ & $\begin{array}{l}\text { VAR. } \\
\text { ACUM }\end{array}$ \\
\hline \multirow{5}{*}{ Resiliencia } & CESM & 0,91 & \multirow{5}{*}{0,83} & \multirow{5}{*}{0,84} & \multirow{5}{*}{0,56} & \multirow{5}{*}{0,78} & \multirow{5}{*}{$62,6 \%$} \\
\hline & CS & 0,74 & & & & & \\
\hline & $\mathrm{AF}$ & 0,86 & & & & & \\
\hline & AS & 0,81 & & & & & \\
\hline & $\mathrm{EP}$ & 0,86 & & & & & \\
\hline \multirow{7}{*}{ Afrontamiento } & FSP & 0,84 & \multirow{7}{*}{0,77} & \multirow{7}{*}{0,85} & \multirow{7}{*}{0,61} & \multirow{7}{*}{0,80} & \multirow{7}{*}{$58,3 \%$} \\
\hline & AFN & 0,79 & & & & & \\
\hline & REP & 0,72 & & & & & \\
\hline & EEA & 0,77 & & & & & \\
\hline & EVIT & 0,71 & & & & & \\
\hline & BAS & 0,78 & & & & & \\
\hline & ESP & 0,81 & & & & & \\
\hline FUFA & & & 0,80 & 0,75 & 0,43 & 0,71 & $56,4 \%$ \\
\hline \multirow{3}{*}{$\mathbf{R A}$} & EXL & 0,86 & \multirow{3}{*}{0,86} & \multirow{3}{*}{0,87} & \multirow{3}{*}{0,52} & \multirow{3}{*}{0,76} & \multirow{3}{*}{$59,1 \%$} \\
\hline & AP & 0,87 & & & & & \\
\hline & SG & 0,86 & & & & & \\
\hline
\end{tabular}

Nota: AFE: Análisis Factorial Exploratorio; F: Factores; CA: Coeficiente alfa; F. RVXG: Factor con rotación Varimax promediado; CVI (global y aceptables) Índices de Validez de Contenido con el método Lawshe; Var Acum.: Varianza acumulada.

Fuente: Elaboración propia a partir de los datos obtenidos de la prueba piloto.

\section{Análisis factorial confirmatorio}

Los indicadores de ajuste del modelo teórico propuesto (cuadro 3), corroboran su adecuación definitiva (bondad de ajuste absoluto), $\mathrm{x}^{2} 340,16$; el ajuste de parsimonia $\mathrm{x}^{2} / \mathrm{gl}$ 0,033, p. $\leq$ 0,000, demuestra un modelo sobreidentificado, apoyada por las medidas incrementales como índice de ajuste comparativo CFI 0,95; TLI 0,95 y RMSEA 0,07 [IC 95\% 0,65-0,80]. Estos datos son soportados por el ajuste de errores estándares SRMS 0,09., en CC.SS. En CC.EE x² 197,820; x²/gl 0,755, p. $\leq$ 0,002; un CFI 0,91; TLI 0,90; RMSEA 0,09 [IC 95\% 0,08-0,10] y SMRS 0,12; estos valores de ajuste muestran un modelo poco satisfactorio; el error de ajuste esperado debe ser menor a 0,10 (Rovira Collado, 2016). El tamaño de las muestras puede explicar estas diferencias (Collado Agudo, 2016; Cupani, 2012). No obstante, el ajuste incremental y de parsimonia, ayudan a soportar el análisis de los factores predictivos en ambos constructos como predictor del RA y el Promedio Ponderado. 
Cuadro 3. Indicadores de Bondad de Ajuste del AFC

\begin{tabular}{|c|c|c|c|c|}
\hline $\begin{array}{c}\text { MEDIDA DE BONDAD DE } \\
\text { AJUSTE }\end{array}$ & $\begin{array}{c}\text { NIVEL DE } \\
\text { AJUSTE } \\
\text { ACEPTABLE }\end{array}$ & $\begin{array}{l}\text { VALORES } \\
\text { OBTENIDOS }\end{array}$ & GL & VALORES \\
\hline \multicolumn{5}{|l|}{ CC.SS } \\
\hline Medida de ajuste absoluto & & 340,160 & & Aceptable \\
\hline Parámetro de no centralidad & & o,366 (p o,o0o) & 97 & Aceptable \\
\hline CFI & $>0,95$ & 0,95 & & Aceptable \\
\hline TLI & $>0,90$ & 0,95 & & Aceptable \\
\hline GFI & $>0,90$ & 0,96 & & Aceptable \\
\hline AGFI & $>0,90$ & 0,92 & & Aceptable \\
\hline RMSEA & $<0,05$ & 0,07 & & Aceptable \\
\hline SRMR & $<0,10$ & 0,09 & & Aceptable \\
\hline \multicolumn{5}{|l|}{ CC.EE } \\
\hline Medida de ajuste absoluto & & 197,820 & & Aceptable \\
\hline $\begin{array}{l}\text { Parámetro de no } \\
\text { centralidad }\end{array}$ & & $0,755(\mathrm{p} 0,000)$ & 97 & Aceptable \\
\hline CFI & $>0,95$ & 0,91 & & Aceptable \\
\hline TLI & $>0,90$ & 0,90 & & Aceptable \\
\hline GFI & $>0,90$ & 0,92 & & Aceptable \\
\hline AGFI & $>0,90$ & 0,90 & & Aceptable \\
\hline RMSEA & $<0,05$ & 0,09 & & Poco satisfactor. \\
\hline SRMR & $<0,10$ & 0,12 & & Poco satisfactor. \\
\hline
\end{tabular}

Nota: X² Chi cuadrado; CFI: Índice de ajuste comparativo; RMSEA: Error cuadrático medio de aproximación; TLI: Índice de Tucker-Lewis; GFI: Índice de bondad de ajuste; SRMR: Raíz media cuadrática estandarizada residual

Fuente: Elaboración propia

Obtener datos robustos, para adaptar los instrumentos y disminuir posibles sesgos en la obtención de información estadística, fue coherente en el presente estudio. El cuadro 4, demuestra que las cargas factoriales de covarianzas/varianzas, los valores van de 0,590 a 0,89 [IC 95\% 0,50-0,95] y son significativas (p. $\leq$ 0,000), para todas las dimensiones de cada instrumento en CC.SS. En CC.EE, los valores, se distribuyen en un rango de 0,55 a o,90 [IC 95\% 0,45-0,97] y son significativos (p. $\leq$ o,000).

Cuadro 4. AFC: Estimación de Cargas factoriales

\begin{tabular}{|c|c|c|c|c|c|c|}
\hline $\begin{array}{l}\text { VARIABLES } \\
\text { LATENTES }\end{array}$ & ESTIMACIÓN & STD.ERR & Z-VAL. & $\mathbf{P}(>|\mathbf{z}|)$ & STD.LV & STD.ALL \\
\hline \multicolumn{7}{|c|}{ CC.SS Estimación de cargas factoriales } \\
\hline \multicolumn{7}{|l|}{ Resi $=\sim$} \\
\hline F 1CESM & 1,00 & & & & 10,39 & 0,87 \\
\hline $\mathrm{F} 2 \mathrm{CS}$ & 0,49 & 0,02 & 17,28 & 0,000 & 5,18 & 0,86 \\
\hline F3AF & 0,41 & 0,02 & 17,65 & 0,000 & 4,26 & 0,89 \\
\hline F4AS & 0,46 & 0,02 & 17,45 & 0,000 & 4,85 & 0,86 \\
\hline F5EP & 0,34 & 0,03 & 17,25 & 0,000 & 3,54 & 0,88 \\
\hline \multicolumn{7}{|l|}{ Afro $=\sim$} \\
\hline F1FSP & 1,000 & & & & 3,781 & 0,89 \\
\hline F2AFNEG & 0,83 & 0,04 & 19,70 & 0,000 & 3,16 & 0,65 \\
\hline F3REVPOS & 0,96 & 0,05 & 21,06 & 0,000 & 3,62 & 0,86 \\
\hline F4EEA & 0,77 & 0,04 & 18,96 & 0,000 & 2,93 & 0,59 \\
\hline F5EEV & 0,97 & 0,04 & 21,26 & 0,000 & 3,69 & 0.87 \\
\hline
\end{tabular}




\begin{tabular}{|c|c|c|c|c|c|c|}
\hline F6BAS & $1, \mathrm{OO}$ & 0,05 & 21,03 & 0,000 & 3,81 & 0,82 \\
\hline F7ESPIRIT & & & & 0,000 & 3,28 & 0,61 \\
\hline$R A=\sim$ & & & & & 4,60 & 0,89 \\
\hline F1EXLOG & 1,000 & & & & 4,60 & 0,89 \\
\hline F2AP.PER & 0,96 & 0,05 & 16,742 & 0,000 & 4,44 & 0,87 \\
\hline F3SGLOB & 0,69 & 0,04 & 16,33 & 0,000 & 3,18 & 0,73 \\
\hline \multicolumn{7}{|l|}{ Covarianzas } \\
\hline \multicolumn{7}{|l|}{$\mathrm{RESI} \sim \sim$} \\
\hline AFRO & 17,43 & 1,01 & 17,23 & 0,000 & 0,44 & 0,44 \\
\hline RA & 29,67 & 1,85 & 15,97 & 0,000 & 0,62 & 0,62 \\
\hline \multicolumn{7}{|l|}{$\mathrm{AFRO} \sim \sim$} \\
\hline $\mathrm{RA}$ & 8,58 & 0,51 & 16,65 & 0.000 & 0,49 & 0,49 \\
\hline \multicolumn{7}{|c|}{ CC.EE Estimación de Cargas Factoriales } \\
\hline \multicolumn{7}{|l|}{ Resi $=\sim$} \\
\hline F1CESM & 1,000 & & & & 6,41 & 0,82 \\
\hline $\mathrm{F} 2 \mathrm{CS}$ & 0,43 & 0,05 & 9,17 & 0,000 & 2,77 & 0,72 \\
\hline F3AF & 0,43 & 0,05 & 9,36 & 0,000 & 2,76 & 0,84 \\
\hline F4AS & 0,32 & 0,04 & 8,18 & 0,000 & 2,05 & 0,53 \\
\hline F5EP & 0,34 & 0,04 & 9,00 & 0,000 & 2,22 & 0,67 \\
\hline \multicolumn{7}{|l|}{ Afro $=\sim$} \\
\hline F1FSP & 1,000 & & & & 2,87 & 0,77 \\
\hline F2AFNEG & 0,94 & 0,13 & 7,29 & 0,000 & 2,70 & 0,53 \\
\hline F3REVPOS & 1,01 & 0,12 & 8,66 & 0,000 & 2,90 & 0,84 \\
\hline F4EEA & 1,05 & 0,14 & 7,41 & 0,000 & 3,02 & 0,54 \\
\hline F5EEV & 1,01 & 0,12 & 8,38 & 0,000 & 2,91 & 0,72 \\
\hline F6BAS & 1,40 & 0,17 & 8,52 & 0,000 & 4,03 & 0,71 \\
\hline F7ESPIRIT & 1,22 & 0,16 & 7,60 & 0,000 & 3,51 & 0,54 \\
\hline \multicolumn{7}{|l|}{$R A=\sim$} \\
\hline F 1EXLO & 1,000 & & & & 3,42 & 0,76 \\
\hline F2AP.PER & 1,12 & 0,14 & 7,82 & 0,000 & 3,85 & 0,90 \\
\hline F3SGLOB & 0,57 & 0,08 & 6,76 & 0,000 & 1,97 & 0,52 \\
\hline \multicolumn{7}{|l|}{ Covarianzas } \\
\hline \multicolumn{7}{|l|}{ Resi $\sim$} \\
\hline Afro & 7,11 & 0,92 & 7,71 & 0,000 & 0,38 & 0,38 \\
\hline RA & 13,87 & 1,72 & 8,04 & 0,000 & 0,63 & 0,63 \\
\hline Afro $\sim \sim$ & & & & 0,000 & & \\
\hline RA & 3,31 & 0,54 & 6,04 & 0,000 & 0,33 & 0,33 \\
\hline
\end{tabular}

Fuente: Elaboración propia.

En el cuadro 5 se presentan los datos del análisis post confirmatorio. El objeto, es, buscar un equilibrio estadístico entre los indicadores de bondad de ajuste del AFC. Para tal efecto, se identificó la fiabilidad compuesta (CF) y el AVE o validez convergente (también denominada varianza extraída promedio). Los parámetros estadísticos evaluados plantean que la confiabilidad compuesta debe ser $>0,07$ y la varianza extraída promedio (AVE) > 0,05 (Aldás y Uriel, 2017). 
Cuadro 5. Análisis Factorial Post Confirmatorio: CR y AVE

\begin{tabular}{|c|c|c|c|c|c|c|}
\hline & \multirow{2}{*}{ ANÁLISIS POST AFC } & \multicolumn{3}{|c|}{ VALOR OBTENIDO } & \multirow{2}{*}{$\begin{array}{c}\text { VALOR } \\
\text { ESPERADO }\end{array}$} & \multirow{2}{*}{$\begin{array}{c}\text { VALIDEZ } \\
\text { CONVERGENTE }\end{array}$} \\
\hline & & Resi & Afro & RA & & \\
\hline \multirow[b]{2}{*}{ CC.SS } & Fiabilidad Compuesta & 0,94 & 0,90 & 0,87 & \multirow[b]{2}{*}{$\mathrm{CR}>0,7$} & \multirow[b]{2}{*}{$\mathrm{AVE}>0,5$} \\
\hline & $\begin{array}{l}\text { Varianza extraída } \\
\text { promedio }\end{array}$ & 0,76 & 0,60 & 0,70 & & \\
\hline \multirow[b]{2}{*}{ CC.EE } & Fiabilidad Compuesta & 0,84 & 0,85 & 0,80 & \multirow[b]{2}{*}{$\mathrm{CR}>0,7$} & \multirow[b]{2}{*}{$\mathrm{AVE}>0,5$} \\
\hline & $\begin{array}{l}\text { Varianza extraída } \\
\text { promedio }\end{array}$ & 0,51 & 0,50 & 0,56 & & \\
\hline
\end{tabular}

Nota: CR Correlación compuesta obtenida > a 0,7. AVE: Análisis de varianza explicada > a 0,5. R.D. Se acepta que los instrumentos son adaptables al contexto de estudio.

Fuente: Elaboración propia.

\subsection{Análisis descriptivo}

En el cuadro 6, en estudiantes de CC.SS los valores promedios de resiliencia $[\mathrm{n}=465]$ es alto en el $58,7 \%$; afrontamiento es bajo en $53,3 \%$; en funcionamiento familiar el $74,4 \%$ es disfuncional; rendimiento académico $52,3 \%$ es bajo, y el promedio ponderado $\geq 14$, considerándose bueno para el contexto nacional. En estudiantes de CC.EE $[\mathrm{n}=131] \mathrm{el}$ $69,4 \%$ es alto en resiliencia; $56,4 \%$ es bajo en estrategias de afrontamiento; en funcionamiento familiar $74,9 \%$ es disfuncional; en rendimiento académico el $59,5 \%$ es bajo; el promedio ponderado es alto $\geq 14$. En análisis de U Mann-Whitney es significativo, corroborándose las diferencias de promedios en cada uno de los constructos en ambos grupos de estudios.

\subsection{Modelo teórico predictivo}

El rendimiento académico (RA), con la irrupción de la teoría social cognitiva y de autoeficacia, está integrada por muchos factores de tipo no cognitivos como: persistencia, motivación, expectativa de logro, aprendizaje percibido y satisfacción global. Estos factores, se asocian a constructos como la resiliencia académica (RESI), afrontamiento (AFRO) y se añade un instrumento de funcionamiento familiar (FUFA), por su papel central en diversos estudios en el Rendimiento académico (RA) (Guardía Olmos, 2016).

Los constructos (latentes), contienen factores susceptibles de ser observados mediante instrumentos psicométricos, con el objeto de hacer plausible su evidencia en entornos académicas universitarios. Factores como: competencia social, confianza en sí mismo, apoyo familiar, apoyo social, estructura personal, pueden direccionar el estilo de afrontamiento como estrategias: focalizado en la solución de los problemas, autofocalización negativa, búsqueda de apoyo social, exposición emocional abierta, evitación, búsqueda de apoyo social, espiritualidad (Guzmán Brito, 2012; Lievens y Sackett, 2011).

Para predecir la variada gama de factores, desarrollamos un modelo explicativo de tipo estadístico y con ello se buscó establecer el valor predictivo de datos ordinales o nominales que representan la percepción de las variables mencionadas, como predictor del rendimiento académico percibido o promedio de notas en un semestre académico (Cabanach et al., 2010; Limonero et al., 2012). 
Cuadro 6. Puntos de corte promedio de las variables

\begin{tabular}{|c|c|c|c|c|c|}
\hline $\begin{array}{l}\text { VARIABLES } \\
\text { LATENTES }\end{array}$ & $\begin{array}{l}\text { PUNTOS DE } \\
\text { CORTE }\end{array}$ & NiVEL & $\%$ & $\bar{x} \quad(\mathbf{s d})$ & $\begin{array}{l}\text { U MANN- } \\
\text { WHITNEY }\end{array}$ \\
\hline \multicolumn{6}{|l|}{ CC.SS } \\
\hline \multirow{2}{*}{ Resi } & Alto: $166-215$ & Alto & $273(58,7 \%)$ & $182,9( \pm 26,9)$ & \multirow{2}{*}{0,000} \\
\hline & Bajo:0-165 & Bajo & $192(42,3 \%)$ & $138,4( \pm 12,0)$ & \\
\hline \multirow{2}{*}{ Afro } & Alto: $135-210$ & Alto & $217(46,7 \%)$ & $157,0( \pm 15,4)$ & \multirow{2}{*}{0,000} \\
\hline & Bajo:0-134 & Bajo & $248(53,3 \%)$ & $115,2( \pm 15,6)$ & \\
\hline \multirow{2}{*}{$\mathrm{FuFa}$} & Func. Fam.O-6 & Buena & $119(25,6 \%)$ & $4,5( \pm 1,6)$ & \multirow{2}{*}{0,000} \\
\hline & Disf. Fam. 7-10 & Inadec. & $346(74,4 \%)$ & $9,1( \pm 1,1)$ & \\
\hline \multirow{2}{*}{ RA } & Alto:87-112 & Alto & $222(47,7 \%)$ & $95,7( \pm 6,7)$ & \multirow{2}{*}{0,000} \\
\hline & Bajo: $0-86$ & Bajo & $243(52,3 \%)$ & $75,8(9,9)$ & \\
\hline \multirow{2}{*}{ PP (PROM) } & Alto $\geq 14$ & Alto & \multirow{2}{*}{$\mathrm{n}=465$} & \multirow{2}{*}{$14.3( \pm 1.26)$} & \\
\hline & Bajo $\leq 14$ & & & & \\
\hline \multicolumn{6}{|l|}{ CC.EE } \\
\hline \multirow{2}{*}{ Resi } & Alto: $166-215$ & Alto & $91(69,4 \%)$ & $182,7( \pm 12,3)$ & \multirow{2}{*}{0,000} \\
\hline & Bajo:0-165 & Bajo & $40(31,6 \%)$ & $153,3( \pm 9,4)$ & \\
\hline \multirow{2}{*}{ Afro } & Alto: $135-210$ & Alto & $57(43,6 \%)$ & $155,3( \pm 19,0)$ & \multirow{2}{*}{0,000} \\
\hline & Bajo:0-134 & Bajo & $74(56,4 \%)$ & $116,4( \pm 13,0)$ & \\
\hline \multirow{2}{*}{$\mathrm{FuFa}$} & Func. Fam.o-6 & Func. & $33(25,15 \%)$ & $5,1( \pm 0,8)$ & \multirow{2}{*}{0,000} \\
\hline & Disf. Fam. 7-10 & Disf. & $98(74,9 \%)$ & $8,8( \pm 1,1)$ & \\
\hline \multirow{2}{*}{ RA } & Alto:87-1 12 & Alto & $53(40,45 \%)$ & $94,3( \pm 6,5)$ & \multirow{2}{*}{0,000} \\
\hline & Bajo: $0-86$ & Bajo & $78(59,55 \%$ & $77,7( \pm 6,3)$ & \\
\hline \multirow{2}{*}{ PP (PROM) } & $\geq 14$ alto & Alto & 131 & $15,29( \pm 1,16)$ & \multirow{2}{*}{0.000} \\
\hline & $\leq 14$ Bajo & & & & \\
\hline
\end{tabular}

Nota: p. 0, OOO $\leq$ 0,05. CC.SS Ciencias de la salud; CC.EE Ciencias empresariales; RA: Percepción de Rendimiento académico; Fufa: Funcionamiento Familiar; Resi: Resiliencia; Afro: Afrontamiento al estrés; PP (PROM) Promedio Ponderado.

Fuente: Elaboración propia.

La figura 1, presenta el modelo explicativo de tipo estadístico que busca evidenciar cómo la resiliencia afecta al rendimiento académico (H1); el afrontamiento afecta directamente el rendimiento académico (H2), la correlación entre la resiliencia y afrontamiento (H3), si afecta la resiliencia afecta el promedio ponderado (H4); el afrontamiento influye directamente en el promedio ponderado (H5) y la influencia directa de rendimiento académico en el promedio ponderado (H6). Se proponen otros modelos específicos. Identificar si los factores psicosociales y sociodemográficos predicen la percepción de rendimiento académico (H7 a H16). Los factores relevantes como funcionamiento familiar, factores de protección y riesgo; factores de afrontamiento activo y pasivo; factores como edad, y especialidad académica también predicen el RA y promedio ponderado (Álvarez y Vernazza, 2017).

\section{Objetivo general: Modelo predictivo general}

En el cuadro 7, el modelo ajustado absoluto en CC.SS es $\mathrm{X}^{2}$ 397,483; ajuste de parsimonia $\mathrm{X}^{2} / \mathrm{gl}$ 0,417/97 los valores son menor de 3 en el parámetro de centralidad. Los indicadores de bondad de ajuste incremental se confirma la predicción de ambos constructos en la percepción de rendimiento académico (CFI 0,095; TLI 0,95; RMSEA 0,08 [IC 95\% 0,04-0,09] y un SRMR 0,953 [IC 95\% 0,800-0,100]. 
Cuadro 7. Indicadores de bondad de ajuste

\begin{tabular}{|c|c|c|c|c|}
\hline MEDIDA DE BONDAD DE AJUSTE & $\begin{array}{c}\text { NIVEL DE } \\
\text { AJUSTE } \\
\text { ACEPTABLE }\end{array}$ & $\begin{array}{c}\text { VALORES } \\
\text { OBTENIDOS }\end{array}$ & GL & VALORES \\
\hline \multicolumn{5}{|l|}{ CC.SS } \\
\hline Medida de ajuste absoluto & & 387,483 & & Aceptable \\
\hline Parámetro de no centralidad & & $0,417(0,000)$ & 97 & Aceptable \\
\hline CFI & $>0,95$ & 0,95 & & Aceptable \\
\hline TLI & $>0,90$ & 0,95 & & Aceptable \\
\hline GFI & $>0,90$ & 0,96 & & Aceptable \\
\hline AGFI & $>0,90$ & 0,94 & & Aceptable \\
\hline RMSEA & $<0,05$ & 0,08 & & Aceptable \\
\hline SRMR & $<0,10$ & 0,09 & & Aceptable \\
\hline \multicolumn{5}{|c|}{ Indicadores de Bondad de ajuste de correlación } \\
\hline Medida de ajuste absoluto & & 387,482 & & Aceptable \\
\hline Parámetro de no centralidad & & $0,417(0,002)$ & 97 & Aceptable \\
\hline CFI & $>0,95$ & 0,95 & & Aceptable \\
\hline TLI & $>0,90$ & 0,94 & & Aceptable \\
\hline GFI & $>0,90$ & 0,90 & & Aceptable \\
\hline AGFI & $>0,90$ & 0,90 & & Aceptable \\
\hline RMSEA & $<0,05$ & $\begin{array}{c}0,08[\mathrm{IC} 95 \% 0,07- \\
0,09]\end{array}$ & & Aceptable \\
\hline SRMR & $<0,10$ & 0,07 & & Aceptable \\
\hline \multicolumn{5}{|l|}{ CC.EE } \\
\hline Medida de ajuste absoluto & & 148,926 & & Aceptable \\
\hline Parámetro de no centralidad & & $569($ p. o,o0o) & 97 & Aceptable \\
\hline CFI & $>0,95$ & 0,65 & & Aceptable \\
\hline TLI & $>0,90$ & 0,56 & & Aceptable \\
\hline GFI & $>0,90$ & 0,90 & & Aceptable \\
\hline AGFI & $>0,90$ & 0,94 & & Aceptable \\
\hline RMSEA & $<0,05$ & $\begin{array}{c}0,06[\mathrm{IC} 95 \% 0,05- \\
0,07]\end{array}$ & & Satisfactorio \\
\hline SRMR & $<0,10$ & 0,07 & & Satisfactorio \\
\hline \multicolumn{5}{|c|}{ Indicadores de Bondad de ajuste de correlación } \\
\hline Medida de ajuste absoluto & & 148,926 & & Aceptable \\
\hline Parámetro de no centralidad & & $0,568(0,001)$ & 97 & Aceptable \\
\hline CFI & $>0,95$ & 0,86 & & Aceptable \\
\hline TLI & $>0,90$ & 0,94 & & Aceptable \\
\hline GFI & $>0,90$ & 0,87 & & Aceptable \\
\hline AGFI & $>0,90$ & 0,86 & & Aceptable \\
\hline RMSEA & $<0,05$ & $\begin{array}{c}0,08[\mathrm{IC} 95 \% 0,07- \\
0,09]\end{array}$ & & Satisfactorio \\
\hline SRMR & $<0,10$ & 0,09 & & Satisfactorio \\
\hline
\end{tabular}

Nota: $\mathrm{X}^{2}$ Chi cuadrado; CFI: Índice de ajuste comparativo; RMSEA: Error cuadrático medio de aproximación; TLI: Índice de Tucker-Lewis; GFI: Índice de bondad de ajuste; SRMR: Raíz media cuadrática estandarizada residual.

Fuente: Elaboración propia.

En CC.EE el RMSEA mejora significativamente 0,064 [IC 95\% 0,050-0,070], pero el indicador CFI 0,65 y TLI 0,56 y un SRMR de 0,178, expresan que el modelo de predicción en CC.SS poco adecuado, aun cuando los datos incrementales son > 0,90. Al evaluar los 
indicadores de ajuste, se observa que los valores son aceptables en CC.SS; sin embargo, en CC.EE, el modelo es mediocre por el valor de SRMR 0,13, cuando lo esperado es hasta 0,10. De acuerdo con estos datos, las diferencias en los indicadores parsimonioso, incrementales y medidas absolutas, tienen valores aceptables y otros variables mediocres, el efecto del tamaño de la muestra condiciona el tipo de resultado.

El cuadro 8, el análisis de regresión confirma el efecto indirecto de la resiliencia hacia el afrontamiento como predictor de rendimiento académico. El valor $\beta 0,48$ es adecuado y la significación $(0,000 \leq 0,05)$ confirman que estos constructos predicen la percepción de rendimiento académico. Es importante destacar que el factor confianza en sí mismo covaría adecuadamente con la capacidad social. Y, los factores de afrontamiento como el apoyo familiar, exposición emocional abierta, espiritualidad y apoyo social covarían adecuadamente para una mejor percepción de sus expectativas de logro, aprendizaje percibido y satisfacción global. En relación con el promedio ponderado no hay significancia y sus $\beta$ son negativas En estudiantes de CC.EE un $B$ o,44 en afrontamiento y B 0,49 en resiliencia; al ser significativas $(0,000 \leq 0,05)$ indican la predicción de ambas en el rendimiento académico. No obstante, los factores de confianza en sí mismo, capacidad social (resiliencia) y la autofocalización emocional abierta con autofocalización negativa covarían con $B$ 0,48 y $B 0,67$ (significativas p. $0,000 \leq 0,05$ ). Se demuestra la importancia de los factores de ambos constructos en la percepción de rendimiento académico; sin embargo, no se observa significatividad con relación al promedio ponderado.

Cuadro 8. Objetivo general: Análisis de regresión

\begin{tabular}{|c|c|c|c|c|c|c|}
\hline $\begin{array}{l}\text { ANÁLISIS DE } \\
\text { REGRESIÓN }\end{array}$ & ESTIMACIÓN & STD.ERR & Z-VAL. & $\mathbf{P}(>|z|)$ & STD.LV & STD.ALL \\
\hline \multicolumn{7}{|l|}{ CC.SS } \\
\hline $\mathrm{PROM} \sim \mathrm{AFRO}$ & $-0,01$ & 0,01 & $-1,09$ & 0,27 & $-0,04$ & $-0,04$ \\
\hline AFRO $\sim$ RESI & 0,18 & 0,01 & 14,30 & 0,000 & 0,48 & 0,48 \\
\hline PROM $\sim$ RESI & $-0,00$ & 0,00 & $-0,03$ & 0,98 & $-0,00$ & $-0,00$ \\
\hline $\mathrm{RA} \sim \mathrm{RESI}$ & 0,22 & 0,02 & 10,16 & 0,000 & 0,49 & 0,49 \\
\hline AFRO & 0,33 & 0,04 & 7,83 & 0,000 & 0,28 & 0,28 \\
\hline $\mathrm{PROM} \sim \mathrm{RA}$ & 0,05 & 0,02 & 2,48 & 0,001 & 0,17 & 0,17 \\
\hline \multicolumn{7}{|l|}{ CC.EE } \\
\hline $\mathrm{PROM} \sim \mathrm{AFRO}$ & 0,01 & 0,02 & 0,47 & 0,640 & 0,03 & 0,03 \\
\hline AFRO $\sim$ RESI & 0,22 & 0,03 & 6,69 & 0,000 & 0,44 & 0,44 \\
\hline PROM $\sim$ RESI & $-0,01$ & 0,02 & $-0,47$ & 0,632 & $-0,07$ & $-0,07$ \\
\hline RA $\sim$ RESI & 0,34 & 0,06 & 5,79 & 0,000 & 0,61 & 0,61 \\
\hline AFRO & 0,11 & 0,08 & 1,40 & 0,162 & 0,10 & 0,10 \\
\hline $\mathrm{PROM} \sim \mathrm{RA}$ & 0,05 & 0,04 & 1,16 & 0,245 & 0,15 & 0,15 \\
\hline
\end{tabular}

Nota: $\beta$ (STD.ALL), Beta; $\mathrm{t}$ (Z-VAL:); Sig. (P(> $|\mathrm{z}|)$

Fuente: Elaboración propia.

La figura 1, se muestra el modelo representativo del análisis de sendero, con el objetivo de graficar cómo los vectores que indican la dirección directa, indirecta y relacional, demuestran la propuesta de estudio relacionada con la predicción del rendimiento académico. Tanto en CC.SS de la salud como en CC.EE, la observancia de los constructos, las estimaciones de la estructura del modelo teórico planteado advierten que existe suficientes evidencias que precisan el patrón predictivo. Es conveniente precisar que si los valores $B$ no están por encima de 0,60 la probabilidad de mejora en el uso de los instrumentos adaptados mediante una validación psicométrica para este escenario incrementaría la significancia de los datos (Rodríguez et al., 2011). 


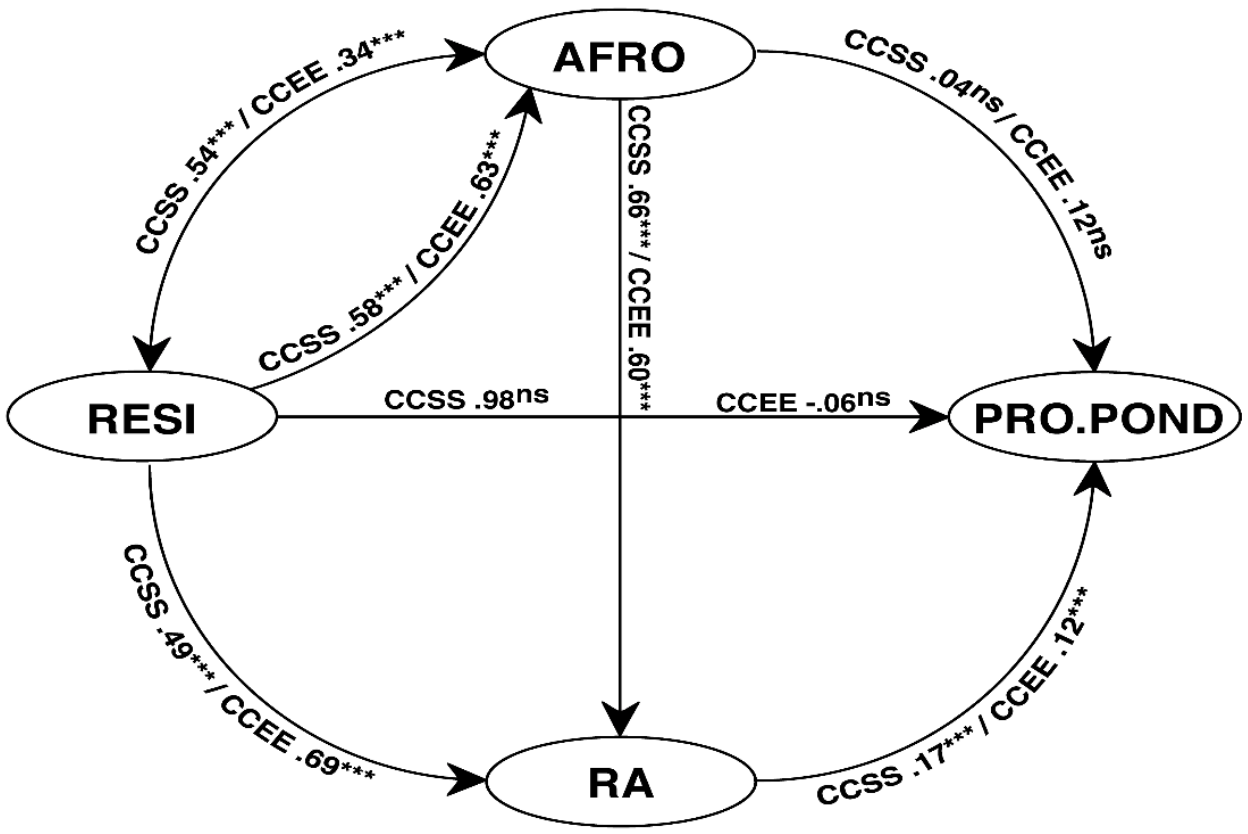

Figura 1. Sendero del modelo predictivo teórico

Fuente: Elaboración propia.

El cuadro 9, en el contraste de hipótesis, los factores de resiliencia como recurso potencial por la experiencia, predice factores de afrontamiento y, éste, predice de manera directa la percepción de rendimiento académico e indirectamente con el afrontamiento (H1 B o,585 $p<0,000 ; \mathrm{H} 2 \mathrm{~B}$ 0,664; $\mathrm{p}<0,000)$. Resiliencia y afrontamiento, no tienen un efecto positivo con el promedio de notas (H3 $\beta-0,044 \mathrm{p}>0,436)$. La percepción de rendimiento sobre el promedio ponderado ( $\mathrm{H} 4 \mathrm{~B} 0,155 \mathrm{p}<0,009)$ en estudiantes de CC.SS no se observa influencia. En CC.EE, resiliencia-afrontamiento tienen efecto directo e indirecto en percepción de RA (H1 B. 0,630 p < 0,000 y H2 ß 0,603 p < 0,000). En Afro hacia promedio ponderado la influencia es no significativa (H3 $B-, 088 \mathrm{p}>0,29)$. RA hacia PP ( 0 o,129 p > 0,246) el efecto no es significativo.

La correlación entre la resiliencia y afrontamiento y su predicción de ambos constructos con la percepción de rendimiento académico en CC.SS, $\beta$ 0,54 (p. 0,000 $\leq 0,05$ ), se afirma que ambos constructos están relacionados y cumplen con predecir la percepción de rendimiento académico. De igual manera en CC.EE un $B .34$ (p. 0,000 $\leq 0,05)$ se cumple la misma predicción, no así con el promedio ponderado. Ambos $\beta$ son negativos y no significativos.

Para estimar mejor la calidad de la predicción tanto de los constructos como de sus factores, se efectuó un análisis de sensibilidad a través de la prueba de bootstrapping para datos de mínimos cuadrados no ponderados. En el cuadro 10, se observa que para el efecto de mediación causal promedio (ACME) de la resiliencia y el afrontamiento en la percepción de rendimiento académico; la propuesta de efectos de promedio directa (ADE); y, las estimaciones de efecto total, como la proporción de efecto mediadora son significativas (p. $0,000 \leq 0,05)$ en la muestra de estudiantes de CC.SS. Sin embargo, en CC.EE., solo es significativa en el efecto promedio directo (ADE) y en el efecto total $(0,000 \leq 0,05)$. No es significativa, en el efecto de mediación de causa y efecto de proporción mediadora (p. 0,340 $\geq 0,05)$. 
Cuadro 9. Contraste de hipótesis

\begin{tabular}{lccc}
\multicolumn{1}{c}{ HIPÓTESIS } & B ESTANDARIZADO & VALOR T & P-VALOR \\
\hline $\boldsymbol{C C} . \boldsymbol{S S}$ : & & & \\
H1: Resi -> RA & 0,49 & 10,16 & $0,000^{*}$ \\
H2: Resi -> Prom & 0,98 & $-0,01$ & 0,980 \\
H3: Afro -> Prom & $-0,04$ & $-0,78$ & 0,430 \\
H4: Resi -> Afro & 0,58 & 14,78 & $0,000^{*}$ \\
H4: Afro -> RA & 0,66 & 17,53 & $0,000^{*}$ \\
H5: RA -> Prom & 0,17 & 2,62 & 0,090 \\
H5: Resi<->Afro->RA & 0,54 & 17,38 & $0,000^{*}$ \\
H6: Resi<->Afro->PROM & 0,86 & 2,23 & $0,002^{*}$ \\
\hline CC.EE & & & \\
H1: Resi -> RA & 0,69 & 5,778 & $0,000^{*}$ \\
H2: Resi -> Prom & $-0,06$ & $-0,47$ & 0,630 \\
H1: Afro -> Prom & $-0,08$ & $-0,08$ & 0,920 \\
H2: Resi -> Afro & 0,63 & 7,22 & $0,000^{*}$ \\
H3: Afro -> RA & 0,60 & 7,15 & $0,000^{*}$ \\
H4: RA -> Prom & 0,12 & 1,16 & 0,024 \\
H5: Resi<->Afro->RA & 0,34 & 6,94 & $0,000^{*}$ \\
H6: Resi<->Afro->PROM & 0,20 & 0,21 & 0,008 \\
\hline
\end{tabular}

Nota: *Significativo (p 0,000 < 0,05).

Fuente: Elaboración propia.

Como el Bootstrapping se orienta a ver la relación en entre las submuestras, es posible generalizar a la población de estudiantes de Ciencias de la Salud; y de manera parcial en estudiantes de Ciencias Empresariales. Estos datos, además, reafirman las diferencias de las muestras $\mathrm{y}$, probablemente la necesidad de utilizar instrumentos con validez psicométrica o, que otras variables no contempladas (espurias) en el presente estudio puedan influir en los resultados obtenidos (Vinod, 2010)

Cuadro 10. Análisis de mediación

\begin{tabular}{|c|c|c|c|c|}
\hline \multirow{2}{*}{ BOOTSTRAP NO PARAMÉTRICO } & \multirow{2}{*}{ ESTIMADO } & \multicolumn{2}{|c|}{ IC $95 \%$} & \multirow[t]{2}{*}{$\overline{\text { P-VALOR }}$} \\
\hline & & L. Inferior & L. Superior & \\
\hline \multicolumn{5}{|l|}{ CC.SS } \\
\hline ACME & 0,04 & 0,02 & 0,06 & 0,000 \\
\hline ADE & 0,21 & 0,17 & 0,25 & 0,000 \\
\hline Efecto Total & 0,25 & 0,22 & 0,29 & 0,000 \\
\hline Propuesta mediadora & 0,16 & 0,10 & 023 & 0,000 \\
\hline \multicolumn{5}{|l|}{ CC.EE } \\
\hline ACME & 0,01 & $-0,01$ & 0,04 & 0,340 \\
\hline $\mathrm{ADE}$ & 0,30 & 0,02 & 0,38 & 0,000 \\
\hline Efecto Total & 0,31 & 0,23 & 0,39 & 0,000 \\
\hline Proporción mediadora & 0,03 & $-0,05$ & 0,34 & 0,340 \\
\hline
\end{tabular}

Nota: p. 0,000 $\leq$ 0,05 para CC. SS; corrobora la predicción de los factores RESI y AFRO en RA. En CC.EE, la mediación de RESI y AFRO tiene efecto promedio directo (ADE) y Efecto total, al ser significativo p. $0,000 \leq 0,05$. No hay efecto causal y la proporción mediadora no son significativas p. $0,340 \geq 0,05$.

Fuente: Elaboración propia. 


\section{Objetivos específicos}

En el Cuadro 11, se presentan los indicadores de bondad tanto para Factores de Riesgo y Protección (FP/FR); Factores de Afrontamiento Activo y Pasivo (AFAC/AFPAS) y Funcionamiento Familiar (FUFA); (nótese que se utilizaron el estimador DWLS y GLS, según sea el caso). Se observa además que, en ambos casos, los valores varían, pero no alteran la naturaleza de los datos. En tanto que, expliquen si los modelos son óptimos, satisfactorios (aceptables) o, mediocres (poco satisfactorio).

Por esa razón, las bondades de ajuste absoluto de factores de protección y riesgo; indicadores incrementales están dentro de los parámetros aceptados en factor de protección, considerándose un modelo satisfactorio en la muestra de CC.SS. Indicadores relacionados con el factor de riesgo son satisfactorios; por ese motivo, aun cuando los indicadores incrementales mejoran el modelo reespecificado, es importante resaltar, que estas diferencias, aun así, pueden afectar el efecto en la percepción de rendimiento académico como en el promedio ponderado. En CC.EE, los indicadores usando el estimador GLS explica adecuadamente el modelo.

En relación con los indicadores de afrontamiento activo, el modelo en ambos grupos de CC.SS y CC.EE los modelos son aceptables en las bondades de ajuste absoluto, incremental y parsimonioso. Respecto del funcionamiento familiar el modelo satisfactorio. El efecto a través de la resiliencia es satisfactorio en la percepción de rendimiento académico. En relación con el promedio ponderado el efecto es mínimo o inexistente. Es decir, en ambas condiciones del entorno familiar afectan directa e indirectamente a la percepción de rendimiento académico en ambos grupos de estudio, a pesar de que el SRMR en la muestra de CC.EE es poco satisfactorio. Sin embargo, los RMSEA están dentro de los parámetros satisfactorios

Cuadro 11. Indicadores de Bondad de Ajuste de factores: P/R; AFAC y AFPAS

\begin{tabular}{|c|c|c|c|c|}
\hline $\begin{array}{l}\text { MEDIDA DE BONDAD } \\
\text { DE AJUSTE }\end{array}$ & $\begin{array}{c}\text { NIVEL DE } \\
\text { AJUSTE } \\
\text { ACEPTABLE }\end{array}$ & VALORES OBTENIDOS & GL & VALORES \\
\hline \multicolumn{5}{|l|}{ CC.SS } \\
\hline \multicolumn{5}{|c|}{ Indicadores de Bondad de ajuste de los Factores Protectores y Riesgo } \\
\hline $\begin{array}{l}\text { Medida de ajuste } \\
\text { absoluto }\end{array}$ & & $346,079 ¥$ & & Aceptable \\
\hline P. de no centralidad & & $0,372(0,000)$ & 97 & Aceptable \\
\hline CFI & $>0,95$ & 0,96 & & Aceptable \\
\hline TLI & $>0,90$ & 0,95 & & Aceptable \\
\hline RMSEA & $<0,05$ & 0,06 [IC 95\% 0,05-0,08] & & Aceptable \\
\hline SRMR & $>0,90$ & 0,08 & & Aceptable \\
\hline \multicolumn{5}{|l|}{ CC.EE } \\
\hline $\begin{array}{l}\text { Medida de ajuste } \\
\text { absoluto }\end{array}$ & $<0,10$ & $134,761 ¥$ & & Aceptable \\
\hline P. de no centralidad & & $0,136(0,041)$ & 97 & Aceptable \\
\hline CFI & $>0,95$ & 0,90 & & Aceptable \\
\hline TLI & $>0,90$ & 0,77 & & Aceptable \\
\hline RMSEA & $<0,05$ & $0,06[$ IC $95 \% 0,05-0,08]$ & & Aceptable \\
\hline SRMR & $>0,90$ & 0,05 & & Óptimo \\
\hline CC.SS & & & & \\
\hline
\end{tabular}

Indicadores de Bondad de ajuste de Afrontamiento Activo y Afrontamiento Pasivo 


\begin{tabular}{|c|c|c|c|c|}
\hline $\begin{array}{l}\text { Medida de ajuste } \\
\text { absoluto }\end{array}$ & & $184,094 \mathrm{p}$ & & Aceptable \\
\hline P. de no centralidad & & $0,198(0,000)$ & 59 & Aceptable \\
\hline CFI & $>0,95$ & 0,96 & & Aceptable \\
\hline TLI & $>0,90$ & 0,95 & & Aceptable \\
\hline RMSEA & $<0,05$ & 0,09 [IC 95\% 0,08-0,10] & & Aceptable \\
\hline SRMR & $>0,90$ & 0,07 & & Óptimo \\
\hline \multicolumn{5}{|l|}{ CC.EE } \\
\hline $\begin{array}{l}\text { Medida de ajuste } \\
\text { absoluto }\end{array}$ & & $136,058 \mathrm{p}$ & & Aceptable \\
\hline P. de no centralidad & & $0,198(0,000)$ & 59 & Aceptable \\
\hline CFI & $>0,95$ & 0,96 & & Aceptable \\
\hline TLI & $>0,90$ & 0,96 & & Aceptable \\
\hline RMSEA & $<0,05$ & 0,06 [IC 95\% $0,05-0,08]$ & & Aceptable \\
\hline SRMR & $>0,90$ & 0,9 & & Satisfactorio \\
\hline
\end{tabular}

\section{CC.SS}

Indicadores de Bondad de ajuste del Funcionamiento Familiar

Medida de ajuste absoluto

P. de no centralidad

CFI $>0,95$

TLI $>0,90$

$<0,05$

RMSEA

$>0,90$

$$
\begin{gathered}
265,544 ¥ \\
0,480(0,000) \\
0,94 \\
0,94
\end{gathered}
$$

0,07 [IC 95\% 0,06-0,08] 0,7

\section{CC.EE.}

Medida de ajuste absoluto

P. de no centralidad

$\begin{array}{ll}\text { CFI } & >0,95 \\ \text { TLI } & >0,90 \\ \text { RMSEA } & <0,05 \\ \text { SRMR } & >0,90\end{array}$

$$
163,218 ¥
$$$$
0,623(0,000)
$$$$
0,90
$$$$
0,90
$$

0,09 [IC 95\% 0,80-0,10]

0,18
Aceptable

94. Aceptable

Aceptable

Aceptable

Aceptable Satisfactorio

Nota: * Diferencias de puntuaciones por uso de estimador DWLS (¥) y GLS (b). Según la variable psicosocial FP/FR y FUFA; En AFAC/AFPAS el estimador GLS (b) para ambas muestras (CC.SS y CC.EE).

Fuente: Elaboración propia.

En la figura 2, los 30,47 del funcionamiento familiar tiene efecto indirecto a través de la resiliencia con el rendimiento académico en CC.SS; el mismo resultado se observa en CC.EE 0,40. En ambos casos es significativo el efecto (p. 0,000 $\leq 0,05$ ). El afrontamiento activo en CC.SS el efecto directo es altamente significativo $\beta$ 0,72. En estudiantes de CC.EE, su efecto es bajo, aunque significativo $(0,000 \leq 0,05)$. El efecto de los factores de protección como de riesgo los $\beta-8,29$ en CC.SS y en CC.EE, $\beta-1,23$ no son significativos en ambos casos. Estos datos adquieren relevancia, es decir, el significado de los factores protectores como: la capacidad social y estructura personal (resiliencia) afectarán a un grupo significativo de la muestra. El cuadro 6, la certeza de disfunción familiar es alta. Tanto en estudiantes de CC.SS $(74,4 \%)$ y CC.EE (74,9\%). La misma proporción de estudiantes de ambos grupos probablemente estarían afectados por los factores de riesgo, ya sea por la falta de apoyo familiar y apoyo social. 


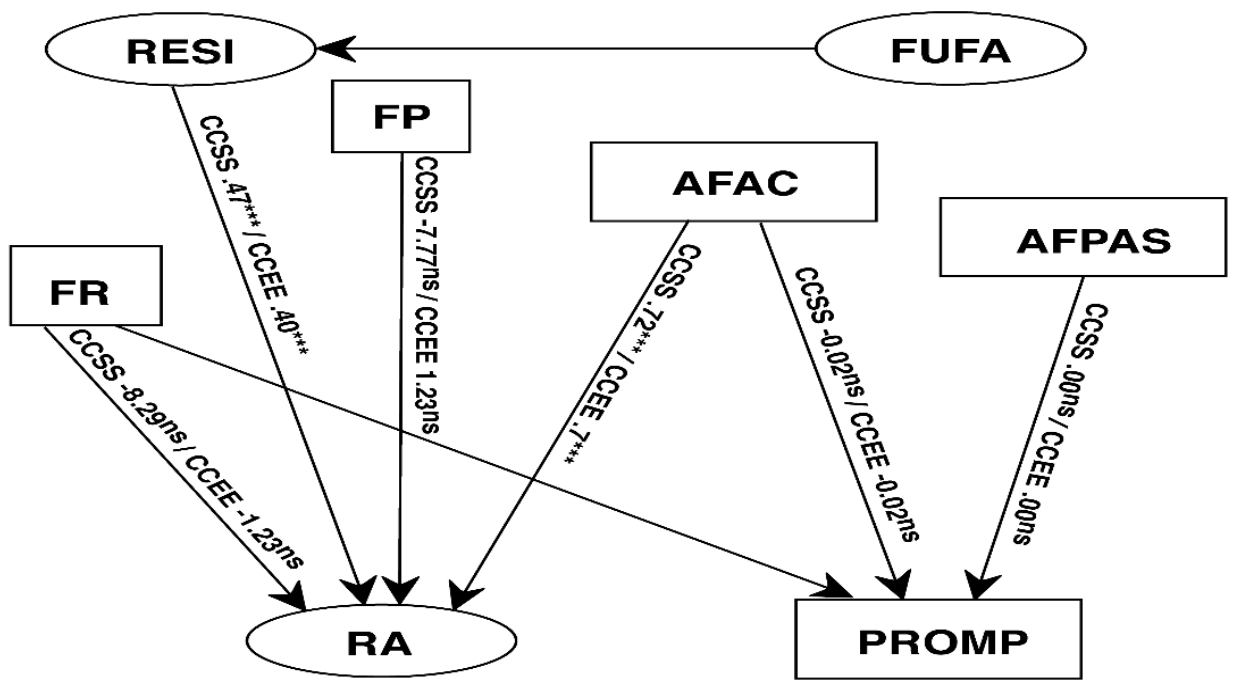

Figura 2. Diagrama de sendero de Factores psicosociales Fuente: Elaboración propia.

Cuadro 12. Contraste de hipótesis de los factores psicosociales

\begin{tabular}{|c|c|c|c|}
\hline HIPÓTESIS & B ESTANDARIZADO & VALOR T & P-VALOR \\
\hline \multicolumn{4}{|l|}{ CC.SS } \\
\hline $\mathrm{H} 7: \mathrm{FP}->\mathrm{RA}$ & 8,291 & 0,76 & 0,440 \\
\hline H8: FP ->PROM & $-3,18$ & $-0,26$ & 0,790 \\
\hline H9: FR->RA & $-7,67$ & $-0,68$ & 0,490 \\
\hline H10:FR->PROM & $-0,02$ & $-0,28$ & 0,790 \\
\hline H11: AFAC $->$ RA & 0,72 & 13,65 & $0,000^{*}$ \\
\hline H12: AFAC -> PROM & $-0,02$ & $-0,14$ & 0,880 \\
\hline H13: AFPAS-> RA & $-0,03$ & $-0,68$ & 0,490 \\
\hline H14: AFPAS-> PROM & $-0,00$ & $-0,05$ & 0,950 \\
\hline H15: FUFA->RESI -> RA & 0,47 & 14,44 & $0,000^{*}$ \\
\hline H16: FUFA ->RESI-> PROM & NA & NA & NA \\
\hline \multicolumn{4}{|l|}{ CC.EE } \\
\hline $\mathrm{H} 7: \mathrm{FP}->\mathrm{RA}$ & 1,23 & 1,93 & $0,005^{*}$ \\
\hline H8: FP ->PROM & 0,07 & 0,13 & 0,890 \\
\hline H9: FR->RA & $-0,61$ & $-0,90$ & 0,360 \\
\hline H10:FR->PROM & $-0,09$ & $-0,21$ & 0,830 \\
\hline H11: AFAC $->$ RA & 0,75 & 7,02 & $0,000^{*}$ \\
\hline H12: AFAC -> PROM & $-0,02$ & $-0,14$ & 0,880 \\
\hline H13: AFPAS-> RA & $-0,03$ & $-0,68$ & 0,490 \\
\hline H14: AFPAS-> PROM & $-0,00$ & $-0,05$ & 0,950 \\
\hline H15: FUFA ->RESI-> RA & 0,40 & 6,76 & $0,000^{*}$ \\
\hline H16: FUFA -> PROM & NA & NA & NA \\
\hline
\end{tabular}

Nota: *Significativo para Afrontamiento activo $(\mathrm{p} 0,000<0,05)$ y significativo para Fufa, Resi, RA (p. 0,000 < 0,05) en ambos grupos. NA: datos no disponibles (no existe efecto en el Pro.Pond.)

Fuente: Elaboración propia.

El cuadro 12 corrobora que un $\beta 0,72(0,000 \leq 0,05)$ que los factores como reevaluación positiva y focalización en la solución de problemas (afrontamiento activo) son los factores claves para dinamizar la eficiencia de los factores protectores como estrategias para afrontar las demandas internas y externas del sistema universitario en grupos de 
estudiantes de CC.SS y en CC.EE. Estas cualidades, influirán de manera esencial en el rendimiento académico percibido, pero no en el promedio ponderado. Otros factores de protección que van a influir son, la confianza en sí mismo, capacidad social y estructura personal. El $\beta 1,23$ positivo, y significativo $(0,05 \leq 0,05)$ tendrán un efecto en el rendimiento académico percibido, pero ese efecto no es sostenible en el tiempo.

\section{Análisis por género y especialidad académica}

Por último, en el análisis de invarianza métrica (cuadro 13) se establece, que la percepción de sexo femenino tiene una leve diferencia comparado con estudiantes de sexo masculino en ambas especialidades. En cambio, lo valores son invariantes según la especialidad relacionado a recurso personales y estilos de afrontamiento y su influencia en percepción de rendimiento académico y promedio ponderado.

Cuadro 13. Análisis de invarianza métrica

\begin{tabular}{|c|c|c|c|c|c|c|c|c|}
\hline & & MODELOS & $\mathrm{X}^{2}$ & GL & CFI & $\Delta \mathrm{CFI}$ & RMSEA & $\Delta$ RMSEA \\
\hline \multirow{8}{*}{ Sexo } & \multirow{4}{*}{ CC.SS } & Configural & 359,70 & 174 & 0,97 & $\mathrm{NA}$ & 0,06 & NA \\
\hline & & Loadings & 369,85 & 186 & 0,97 & 0,000 & 0,06 & 0,003 \\
\hline & & Escalar & 380,77 & 198 & 0,97 & 0,000 & 0,06 & 0.002 \\
\hline & & Means & 393,11 & 201 & 0,97 & 0,002 & 0,06 & 0,001 \\
\hline & \multirow{4}{*}{ CC.EE } & Configural & 357,24 & 174 & 0,97 & $\mathrm{NA}$ & 0,06 & $\mathrm{NA}$ \\
\hline & & Loadings & 375,07 & 186 & 0,97 & 0,001 & 0,06 & 0,002 \\
\hline & & Escalar & 382,89 & 198 & 0,97 & 0,001 & 0,06 & 0,003 \\
\hline & & Métrica & 401,87 & 201 & 0,96 & 0,003 & 0,06 & 0,000 \\
\hline \multirow{8}{*}{$\begin{array}{l}\text { Especialidad } \\
\text { Académica }\end{array}$} & \multirow{4}{*}{ CC.SS } & Configural & 388,96 & 261 & 0,98 & $\mathrm{NA}$ & 0,05 & $\mathrm{NA}$ \\
\hline & & Loadings & 435,30 & 285 & 0,97 & 0,002 & 0,05 & 0,000 \\
\hline & & Escalar & 439,28 & 309 & 0,98 & 0,002 & 0,05 & 0,004 \\
\hline & & Métrica & 462,80 & 315 & 0,97 & 0,003 & 0,05 & 0,003 \\
\hline & \multirow{4}{*}{ CC.EE } & Configural & 275,70 & 261 & 0,98 & NA & 0,03 & NA \\
\hline & & Loadings & 327,33 & 285 & 0,98 & 0,021 & 0,05 & 0,023 \\
\hline & & Escalar & 341,08 & 309 & 0,97 & 0.008 & 0,04 & 0,010 \\
\hline & & Métrica & 347,53 & 315 & 0,97 & 0,000 & 0,04 & 0,000 \\
\hline
\end{tabular}

Nota: NA: Datos no disponibles.

Fuente: Elaboración propia.

\section{Discusión y conclusiones}

Las investigaciones de la resiliencia y afrontamiento tradicionalmente se han relacionado con factores psicosociales, sociodemográfica, infraestructura, académicos y de procesos académicos, didáctica universitaria y su evaluación con las calificaciones semestrales o finales de un periodo lectivo anual. Sin embargo, establecer si estos factores tienen relevancia en la percepción del rendimiento y su repercusión en el resultado de ese proceso, es casi escaso en la comunidad universitaria peruana (Aquino Guillen, 2016; Pizarro Cherre, 2017).

La necesidad de realizar estudios predictivos que observen influencias o efectos directos, indirectos, mediacionales y relacionales entre estos constructos (resiliencia y afrontamiento) con la percepción de rendimiento académico como parte del proceso de enseñanza-aprendizaje y su enlace con la nota promedio (entendida como resultado), demandó la necesidad de un objetivo central, establecer si los factores de ambos constructos son predictores del rendimiento académico en el proceso (percepción de 
rendimiento) y su resultado (la nota promedio ponderada). Medrano (2010) plantea la necesidad de desarrollar estos estudios ante la falta de predecir si las creencias, expectativas, percepción de aprendizaje y satisfacción tienen un efecto directo, indirecto, relacional o moderador de estos procesos. Estos son inherentes a la dinámica académica de la vida universitaria. Postula la idea que la autoeficacia tiene que ver con aquellas creencias académicas para obtener buenas notas. Es decir, plantea realizar estudios en la que los patrones no cognitivos por sí mismo impactan en el rendimiento o, en su defecto, los patrones no cognitivos (actitudes, creencias, expectativas de logro, aprendizaje percibido, entre otros) contribuyen a que los patrones cognitivos (atención, memoria, pensamientos procesuales, atención) garantizarán el logro académico esperado por el estudiante y la universidad según su declaración intencional, soportado por la estructura académica y la infraestructura actual (González y Landero, 2007).

El tema recurrente está entonces, en que los diseños de licenciamiento y acreditación nacional contempla y demanda el desarrollo de perfiles del ingresante, del egresado y las universidades recién en estos últimos 5 años vienen implementando ese modelo de proceso y ahora variaron a el modelo de resultado sin establecer cuáles en sí son esos perfiles claves para garantizar la calidad académica (Sineace, 2016)

Diferentes estudios en otros contextos tienen ya, una tradición en la búsqueda de esos patrones no cognitivos de tipo metamotivacionales (resiliencia) y metaemocionales (afrontamiento), demostrándose que existe un fuerte impacto predictivo en el rendimiento académico de procesos (percepción de rendimiento) y su influencia es mínima en el promedio de notas. Se han considerado variables como la edad, sexo, dinámica familiar, estructura personal, social, creencias y expectativas, capacidad autorregulatoria, así como la motivación, actitudes, flexibilidad cognitiva, autoeficacia cognitiva, flotabilidad académica (bouyance academic). Factores que contribuyen en el estudiante al manejo de las incertidumbres y la búsqueda de certezas para su éxito académico (Datu y Yuen, 2018; Martin et al., 2013).

En la década del noventa la teoría sociocognitiva y de autoeficacia de Bandura (Zimmerman, Bandura y Martínes-Pons, 1992) había postulado que los factores no cognitivos como las expectativas de logro, creencias asociadas a su aprendizaje, el estilo de percibir su aprendizaje, la búsqueda de autonomías como el tener control sobre sus propias actividades académicas. Incluso, se han incorporado otros constructos no cognitivos como: la automotivación, la capacidad social, la estructura personal, experiencias vivenciales, estilos emocionales, capacidad de decisión, resolución de problemas impactan en los dominios (aprendizajes por competencias) y, estos repercuten en el logro académico deseado como al buen desempeño académico y convivencia universitaria (Farnos Miró, 2010, 2013; Lamas, 2015; Medrano, 2011).

Estos presupuestos teóricos son los que guían el presente estudio. Como se puede observar, fue conveniente y necesario determinar una muestra representativa de estudiantes universitarios (en este caso estudiantes de ciencias de la salud: medicina, enfermería y tecnología médica) y, compararlos con estudiantes de ciencias empresariales (ingeniería de sistemas empresariales, ingeniería económica y administración de negocios internacionales). La selección al muestreo estratificado fue un problema crítico a superar sobre todo en la muestra de las carreras de gestión por el número de la muestra $[\mathrm{n}=131]$. En cambio, sí se logró tener una muestra mayor en estudiantes de ciencias de la salud [n $=465]$. Otro factor crítico se relacionó con los instrumentos seleccionados para medir los 
constructos de resiliencia y afrontamiento (Resi-M, Palomar y Gomez, 2010; CAE, Sandín y Chorot, 2003); además de adecuar tres dimensiones de dos instrumentos en la percepción de rendimiento académico. Dimensión expectativa de logro y aprendizaje percibido (Medrano, 2011), dimensión satisfacción global (Medrano, Laporice y Pérez, 2014).

Con la finalidad de adecuar estos instrumentos en el cuadro 1, los resultados obtenidos de las pruebas de adecuación a la muestra para factorizar el constructo como una variable eficiente (KMO) de igual manera la prueba de Bartlett para demostrar que las muestras provienen de las poblaciones de estudiantes seleccionadas resultó ser significativa. Cumplido este requisito, no fue suficiente adaptar los instrumentos a la muestra recurriéndose al análisis factorial exploratorio (AFE) y con el objeto de no modificar las escalas y sus dimensiones se realizaron las pruebas de fiabilidad por dimensiones y de manera global y se promedió los factores rotados Varimax vinculándose además con el coeficiente de validez de contenido global y aceptables. También, evaluar las varianzas acumuladas; todos estos datos resultaron satisfactorios por sus valores aceptables para la fiabilidad, los promedios factores rotados y las varianzas extraídas mediante el criterio de Moriyama para criterio de jueces. Estos valores fueron cercanos a los obtenidos por los autores originales, con la única a excepción de la escala de percepción de rendimiento académico, a pesar de ello, su valor estadístico obtenido, también se encontró dentro de los valores esperados (cuadro 2).

Cumplidos estos criterios estadísticos fue pertinente realizar el análisis factorial confirmatorio (AFC), los valores de ajuste absoluto, incremental y de parsimonia están dentro de lo esperado en CC.Ss, excepto que el RMSEA y SRMR son valores no esperados, por lo que se atribuye como valores poco satisfactorios en la muestra de estudiantes de CC.EE (cuadro 3). Pero, al analizar la estimación de las cargas factoriales (cuadro 4), los factores se encuentran por encima de 0,06 a 0,09 en CC.SS, y de 0,05 a 0,08 en CC.EE. Y, en ambos casos, son significativos. En este mismo cuadro 4, en ambas muestras las covarianzas son significativas con valores aceptables. Admitir estos valores no son suficientes. El propósito, era ir más allá con la robustez estadística exigida. Se efectuó un post análisis factorial confirmatorio. Se evalúan las cargas factoriales para la muestra de CC.SS y de CC.EE (cuadro 5), estas cargas están por encima de 0,07 a 0,09 y lo esperado era superior a siete. Además, se añadió un segundo tipo de validez, la validez convergente, obteniéndose datos superiores a 0,05 que era lo esperado (Aldás y Uriel, 2017; Cabello y Chirinos, 2012; Escobedo et al., 2016; Vinod, 2010).

Logrado la determinación de dotar de un cierto grado de robustez estadística, se aplicaron los instrumentos a la muestra estratificada (cuadro 5), mediante el método automático de categorización, los puntajes para cada uno de los constructos. En CC.SS se encuentran valores porcentuales en resiliencia de 58,7\% alto; afrontamiento 53,3\% bajo; $74,4 \%$ de disfuncionalidad familiar; percepción de rendimiento académico 52,3\% bajo y Promedio ponderado superior 14, estimándose como un valor cuantitativo bueno a alto.

En estudiantes de CC.EE, una resiliencia de $69.4 \%$ es alto; afrontamiento $56,4 \%$ bajo; el funcionamiento familiar $74,9 \%$ es altamente disfuncional. La percepción de rendimiento académico $59,5 \%$ bajo. El promedio ponderado mayor a 14 (es importante destacar que en CC.EE, la norma aprobatoria es de 13; a diferencia de CC.SS, que el promedio aprobatorio es 11). La prueba de U Mann-Whitney al ser significativa, refleja que los valores de las dos muestras tienen patrones diferentes en su perfil académico; esta diferencia, repercutirá significativamente en la percepción de rendimiento y también, en el promedio ponderado. 
Además, se infiere que los perfiles personales, podrían influir en sus patrones no cognitivos y cognitivos.

Según los resultados obtenidos, la adecuación de las muestras a la población y la adaptación (parcial) de los instrumentos para medir los constructos, nos permitió describir un modelo teórico estadístico general y específico (figuras 1 y 2), en la que se proponen hipótesis de trabajo centrales. La resiliencia predice de manera directa e indirecta el rendimiento académico en ambas muestras de estudiantes universitarios; el afrontamiento tiene una influencia directa sobre el rendimiento académico y cundo se correlaciona con la resiliencia resulta ser significativa. Por otro lado, tanto la resiliencia como el afrontamiento tiene influencia mínima o inexistente con el promedio ponderado. Tal como se observa en el cuadro 7 , los indicadores de bondad de ajuste absoluto, incremental y de parsimonia (RMSEA 0,08 [IC 95\% 0,07-0,09], el SRMR es 0,07 en CC.SS.

Los estudiantes de CC.EE, también es satisfactorio el RMSEA 0,09 [IC 95\% 0,07-0,09], el SRMR 0,09. Estos datos, son considerados por algunos autores como modelos satisfactorios, al observarse valores de 0,10 . En cambio, otros consideran óptimo un RMSEA inferior a 0,05. (Collado Agudo, 2016; Cupani, 2012; Piemontesi, Heredia y Furlán, 2012). Estas diferencias en la forma de interpretación requieren de un análisis más exhaustivo para consensuar criterios de valoración para futuros estudios.

Si evaluamos los resultados en el mismo cuadro 7 , la relación entre ambas variables, los valores hallados van de un RMSEA 0,08 [IC \% 0,07-0,09] y SRMR 0,07 en CC.SS., y un RMSEA 0,08 [IC 95\% 0,07-0,09] SRMR 0,09 en CC.EE. Por consiguiente, se establece que, para este estudio, la resiliencia si predice la percepción de rendimiento académico. Al relacionar ambos constructos también, predece el rendimiento académico. Lo que si queda claramente demostrados es que no existen valores significativos para el promedio ponderado como se observará en la figura 1 del modelo predictivo.

Para consolidar la afirmación de estos datos encontrados, los indicadores de bondad de ajuste en el cuadro 8, muestran que los valores del análisis de regresión confirman que, la resiliencia a través del afrontamiento predice la percepción de rendimiento académico. El efecto de la resiliencia es positivo y significativo en resiliencia y afrontamiento (vía indirecta); 0,48 (0,000 $\leq 0,05) ; 0,49(0,000 \leq 0,05)$; vía directa; afrontamiento vía directa con el RA es positivo 0,28 y significativo $(0,000 \leq 0,05)$. Un valor positivo y significativo entre RA y PROM 0,17 $(0,000 \leq 0,05)$ confirma los datos de algunos autores, que existe en algunos casos un mínimo efecto (Rodríguez Ayán y Ruíz Díaz, 2011).

Es obvio que en otros casos se han encontrado que la relación con los valores de resiliencia y afrontamiento con el promedio ponderado, son valores negativos y no significativos. Estos hallazgos corroboran que la resiliencia y afrontamiento predicen el rendimiento académico como un proceso; más no, como un resultado -promedio ponderado- (Gaxiola et al., 2012; López-Cortón, 2015; Torrecillas et al., 2016).

La figura 1, se observa que los valores citados son mejores en sus cargas, para RESI-RA $B$ 0,58 y significativo en CC. SS; $\beta$ 0,63 significativo. En CC.EE, al correlacionar RESI y AFRO RA $\beta 0,54$ y $\beta 0,34$, es significativo, estos datos confirman la misma tendencia.

Es obvio que cuando se analiza la vía directa e indirecta de RESI-AFRO con rendimiento académico, $\beta$ 0,65 y $\beta$ o,60 en ambas muestras no hay efecto sobre el promedio ponderado. Pero, los $\mathrm{B} 0,17$ y 0,12 son significativos entre rendimiento académico y promedio ponderado (cuadro 9). Este hallazgo confirma de alguna manera la existencia de alguna 
influencia de los constructos (RESI y AFRO) pero es muy débil. En el contraste de hipótesis se confirma la tendencia de los datos con otros estudios en culturas distintas (Limonero et al., 2012; Minulescu, 2015; So-Hee y Sang-Sook, 2016).

Finalmente, para probar nuestro modelo general de influencia directa, indirecta (mediacional) y relacional, se realizó una prueba de Bootstrapping (o análisis de medición) (cuadro 10). La confirmación de la predicción es valiosa. Los valores son positivos y significativos, confirmándose la alta probabilidad que hay predicción de constructos de en el rendimiento académico, pero como proceso (percepción). Y, es mínima o inexistente, en el promedio ponderado. Además, esta prueba, nos indica que es posible generalizar los datos alcanzados en ambas poblaciones de las dos universidades privadas estudiadas.

Ahora, cuando se intenta evaluar los factores predictores de la resiliencia y el afrontamiento con la percepción de rendimiento académico y promedio ponderado, en el cuadro 11, los hallazgos en este estudio revelan que los indicadores de los factores protectores y factores de riesgo; los indicadores de bondad de ajuste con satisfactorios en CC.SS, RMSEA 0,06 [IC 95\% 0,05-0,07] y SRMR 0,08. El RMSEA 0,06 [IC 95\% 0,05-0,07] es apoyado por el SRMR $0,13>$ a 0,05 en CC.EE, resulta un modelo poco satisfactorio a diferencia de la muestra de estudiantes de CC.Ss. Respecto de los factores de Afrontamiento activo y Afrontamiento pasivo, las bondades de ajuste absoluto, incremental y parsimonia son aceptables, así como el RMSEA 0,09 [IC 95\% 0,08-0,10] y SRMR 0,09 son satisfactorios para CC.SS y un RMSEA 0,08 [IC 95\% 0,07-0,09] también se considera satisfactorios. En Funcionamiento Familiar los indicadores de bondad de ajuste aceptables y RMSEA 0,08 [IC 95\% 0,07-0,09], SRMR 0,09 para CC.SS; RMSEA 0,07 [IC 95\% 0,06-0,08] y un SRMR 0,09 es satisfactorio. Pero los indicadores de bondad de ajuste al ser aceptables para CC.EE, el RMSEA 0,09 [IC 95\% 0,08-0,10]; pero el SRMR es de 0,18>0,10, datos que no garantizan lo satisfactorio del modelo aun cuando los indicadores reflejan ciertos valores aceptables.

La figura 2, demuestra que los valores de Funcionamiento familiar por vía indirecta (resiliencia) hacia el RA, un $B \quad 0,47$ y $B \quad 0,40$ positivos y significativos (tanto para CC.Ss y CC.EE) prueba que el factor familiar es cardinal para la vida académica universitaria. Particularmente en la percepción de rendimiento académico, y si, relacionamos la disfunción familiar de ambas muestras ratifica la baja sostenibilidad de los recursos a pesar de haber indicadores satisfactorios y óptimos tanto en el objetivo general como los específicos.

Estos datos pueden explican problemas futuros para un adecuado desempeño académico de calidad. El cuadro 12, valores $B-7,77$ y $-1,23$ (CC.SS y CC.EE respectivamente), los factores protectores, no garantizan que los estudiantes estén empleando todas sus potencialidades en diario quehacer universitario. Respecto de los factores de riesgo la tendencia de un $\beta$ no positivo (-8,28 y -0,61), y no significativo (p. 0,36 > 0,05), no tienen valor predictivo para la percepción de rendimiento académico y el Promedio Ponderado.

No obstante, en afrontamiento activo $\beta$ O,72 en CC.SS y, en CC.EE, $\beta$ O,70 al ser positivos y significativos (p. $0,000<0,05)$, ajustarían la capacidad adaptativa para dinamizar los factores de protección. No obstante, la presencia de disfunción familiar en ambos grupos de estudiantes no permite la sostenibilidad de estas estrategias para manejar y autorregular adecuadamente las actividades diarias de la vida académica universitaria. Factores de, confianza en sí mismo y calidad personal como la estructura personal pueden predecir una mejor percepción de rendimiento académico cuando el factor familiar emplea una vía indirecta (o mediadora) a través de la resiliencia y mejorar la percepción de 
rendimiento académico (Díaz y Arancibia, 2002). Por otro lado, factores de reevaluación positiva, focalización en la solución de problemas, son factores significativos en la vida académica más en estudiantes de ciencias de la salud que en estudiantes de ciencias empresariales. Observaciones en distintos estudios la autoevaluación, reevaluación de sus recursos, búsqueda de apoyo social, búsqueda del sentido del humor y satisfacción, espiritualidad (Adebisi Fayombo, 2011; Álvarez y Vernazza, 2013; Becerra y Morales Ballesteros, 2015; Cassaretto y Martínez, 2009; Hernández y Roviera Millán, 2016), constituyen recursos alternativos para evitar una de las principales causas de deserción universitaria.

Los reportes de varias investigaciones concluyen que, problemas familiares, síntomas depresivos, problemas de atención, pensamientos y creencias negativas recurrentes, agudizan la baja eficiencia en el empleo de recursos y la creación de nuevos estilos sostenibles para afrontar adecuadamente los acontecimientos propios de la actividad académica (Lugo-Márquez et al., 2016). Por este motivo es que el afrontamiento activo, sostiene mejor la habilidad de los estudiantes para afrontar situaciones de conflicto; estás habilidades se apoyan también en los factores de focalización en la solución de problemas y reevaluación positiva de las emociones (Gayles, 2003; Villalta Paúcar, 2010)

En relación con los factores sociodemográficos, la invarianza métrica (cuadro 13), se observa que las mujeres, tienen una diferencia de percepción leve con relación a sus potencialidades y talentos que los varones. No se encuentra influencia determinante de la especialidad académica. Estos datos se confirman cuando los estudiantes, al desarrollar confianza en sí mismo, utilizar estrategias de autofocalización negativa y conductas evitativas, búsqueda de la calidad del apoyo social no garantizan la eficiencia cognitiva y se refleja en sus acciones académicas y personales (Gómez-Collado, 2012; Sanz Oro, 2010).

También, los factores de capacidad social, confianza en sí mismo, sentirse motivados para aprender estrategias de autoeficacia (Luthar, Cicchetti y Becker, 2000), con subsidiarias de una mayor demanda del sistema universitario para mejorar la estructura personal de los futuros ingresantes, mediante perfiles de ingreso y durante toda la vida formativa. Ello garantiza las expectativas de logro y perciban mejor su aprendizaje, desarrollando políticas educativas proactivas. Los estudiantes, aprenden de alguna manera u otra a manejar adecuadamente el estrés (Gonzáles y Landero, 2007). La confianza en que estos factores al ser promovidos adecuadamente por los programas académicos definitivamente mejoraran el patrón emocional de los estudiantes y su impacto en sus procesos cognitivos y no cognitivos para un desarrollo académico (Becerra y Morales Ballesteros, 2015; Canales y De los Ríos, 2007).

En consecuencia, el logro académico de los estudiantes de ciencias de la salud y en alguna medida de los estudiantes de ciencias empresariales; a lo largo de este estudio genera certidumbre en que, la capacidad de logro académico puede motivar la mejora de resultados de proceso (percepción de rendimiento académico) y, contribuir a mejores resultados cuantitativos con el promedio ponderado semestral o anual (Lamas, 2015; Medrano, 2011).

Por otro lado, la necesidad de evaluar a los estudiantes respecto de estos factores no cognitivos planteados estaría enfocados a impactar en el estudiante tanto en el perfilamiento personal emocional, social, como cognitivo (Cano García y Fernández Ferrer, 2015). Además, de la posibilidad de crear vínculos de trabajo integrado, desarrollar acciones de colaboración y cooperación para el trabajo en equipo, influenciar en el 
estudiante a vincularse mejor con la universidad para "despertar" sus potencialidades que muchas veces (por la experiencia docente) no son convenientemente exploradas por el sistema universitario (Alvaréz Botello et al., 2015; Martin y Marsh, 2009; Rovira-Collado, 2016). Modelar dominios orientados a desarrollar perfiles de estudiantes para mejorar y asegurar la calidad de la vida académica universitaria es pertinente es una necesidad en las actuales circunstancias, productos de los profundos cambios en el contexto universitario (Monacis et al., 2014).

En conclusión, La resiliencia de manera directa y a través del afrontamiento (indirecta o mediacional), predicen la percepción de rendimiento académico. También, el afrontamiento tiene un impacto directo en el rendimiento académico y cuando se correlacionan entre la resiliencia y el afrontamiento se consolidan los supuestos previos de identificar el valor de los factores de ambos constructos. El impacto predictivo en el promedio ponderado no es significativo; por lo que, se hace necesario asociar nuevas variables para un análisis más profundo del problema. Además, en los modelos psicosociales como es el funcionamiento familiar y los factores protectores y afrontamiento activo y pasivo, se encuentra que el funcionamiento familiar, es un buen predictor del rendimiento académico. Sobre todo, cuando se asocia a factores resilientes como confianza en sí mismo, calidad social y una buena estructura personal.

Los factores protectores y de riesgo, no tienen un efecto directo e indirecto en el rendimiento académico, aun cuando otros estudios consideran que el rol de los factores protectores (confianza en sí mismo, calidad social, estructura personal) son relevantes para el desarrollo académico del estudiante. Factores de afrontamiento activo (focalización en la solución de problemas, la reevaluación positiva de la situación personal y del entorno), son factores de garantía para la resiliencia y el afrontamiento en la predicción del rendimiento académico por procesos (percepción de rendimiento). Aun así, es conveniente realizar nuevos estudios que incorporaren variables como el ambiente académico, la infraestructura, la dinámica docente entre otros factores, para mejorar la predicción de estos constructos.

\section{Limitaciones del estudio y proyecciones futuras}

Una de las limitaciones del presente estudio está ligada al tipo de muestreo complejo como es el estratificado. Esta complejidad, implica definir convenientemente reglas de muestreo, es decir, la homogenización y ponderación muestral para cada estrato seleccionado. Es propicio, que el tamaño de la muestra en general debe estar ligado al número de ítems de cada dimensión o factor, los ratios de observaciones adecuadas al objetivo de la investigación y, la relación a la definición esencial de los constructos o modelo teórico elegido. Otra limitación sobresaliente, es el correlato entre estos muestreos complejos y la adopción de los instrumentos. Si tenemos en cuenta el número de ítems y el tamaño de la muestra debe ser suficiente.

Por tal motivo sus dimensiones o factores deben estar convenientemente asociados con la fiabilidad compuesta. El objeto es, poder realizar análisis completos y no parciales de los valores obtenidos. También, es inevitable tener en cuenta la validez convergente con el propósito de correlacionar fuertemente los ítems a las dimensiones de análisis no comprometiendo el valor de cada indicador del instrumento (Álvarez y Vernazza, 2017; Frías-Navarro y Soler, 2012). El tener una correspondencia adecuada en el control del grado de correlación con las hipótesis planteadas, las variables latentes y su conexión estructural, es clave en estos estudios con SEM. Con esta acción, se evitaría inducción a 
errores, al poner en cuestión la adaptación de los cuestionarios con la capacidad discriminante de cada uno de ellos. Por último, se puede realizar la validez nomológica, para correlacionar valores obtenidos con el constructo teórico, enlazarlos con otros constructos y sus respectivos cuestionarios como es este caso. Aspectos, que solo se pudo resolver con los tres primeros presupuestos: no alterar la naturaleza original de las dimensiones de los instrumentos relacionados con los tres constructos, realizar una fiabilidad compuesta, promediar los factores rotados para (aparentemente minimizar el número de variables), considerar los valores del KMO, la prueba de Bartlett y la significación, además de añadir la varianza acumulada extraída. Sin embargo, la consideramos insuficientes (Vinod, 2010; Yamamoto, 2017)

En investigaciones Futuras, es recomendable contemplar las guías de muestreo y selección de instrumentos, no solo para adaptarlos a una realidad distinta de donde fue elaborada. El asunto es, asegurar una alta validación y aplicabilidad transcultural, facilitar su extrapolación a poblaciones mayores en el mismo contexto de estudio o, a otras instituciones, que se encuentran con la misma exigencia de hallar perfiles de estudiantes con capacidades resilientes y empleo de estrategias de afrontamiento. La oportuna y adecuada acción en las medidas y estructuras de los instrumentos, ayudará ostensiblemente a desarrollar programas de intervención temprana, como las tutorías presenciales y virtuales.

Esta tendencia, es necesaria en un panorama global, donde el estudiante, es el foco central de su aprendizaje. Entonces, se necesitan protocolos y guías de calidad en la evaluación previa a la intervención docente, esta es, evitar un mal mayor, la alta deserción universitaria en el país. Cumplidos estos presupuestos, como promover el desarrollo de potencialidades, capacidades creativas, innovadoras y emprendedoras en cada carrera profesional; los estudios causales, deben asumir la rigurosidad de la selección muestral compleja y hallar la validez psicométrica, vinculado al manual de intervención docente para lograr personas resilientes, potenciar estrategias protectoras, mejorar los afrontamiento activos, fomentar expectativas de logro, mejorar la percepción de sus aprendizajes para un bien mayor. Es decir, la satisfacción global en el plano institucional y personal del estudiante.

\section{Agradecimientos}

Agradecer el apoyo de la Enfermera Marina del C. Menchola, Ingenieros: Alberto Victoria, Helga y Valeria Tipismana; además de la motivación y guía de los Mg. Fernando Muñoz, Master on Art, Health Education Fernando Runzer Colmenares y al Dr. Juan Torres, para llevar un estudio poco realizado en universidades peruanas, a pesar de su enorme necesidad para garantizar el aseguramiento de la calidad educativa. Además, de la enorme satisfacción de desarrollar un estudio, que cumpla con los rigores de una maestría en educación superior con mención en docencia e investigación universitaria.

\section{Referencias}

Adebisi Fayombo, G. (2011). Student-related variables as predictors of academic achievement among some undergraduate psychology students in Barbados. US-China Education Review, 23, 280-289.

Aldás, J. y Uriel, E. (2017). Análisis multivariante aplicado con R. Madrid: Ediciones Paraninfo. 
Álvarez Botello, J., Chaparro Salinas, E. y Reyes Pérez, D. (2015). Estudio de la satisfacción de los estudiantes con los servicios educativos brindados por instituciones de educación superior del Valle de Toluca. REICE. Revista Iberoamericana sobre Calidad, Eficacia y Cambio en Educación, 13(2), 5-26.

Álvarez, R. y Vernazza, E. (2013). Aplicación de los modelos de ecuaciones estructurales para el estudio de la satisfacción estudiantil en los cursos superiores de FCCEEyA. Montevideo: Instituto de Estadística.

Álvarez, R. y Vernazza, E. (2017). Evaluación de un instrumento de medición del nivel de satisfacción estudiantil a través de la aplicación de modelos de ecuaciones estructurales. Cuadernos del CIMBAGE, 19, 1-25.

Antal, E. y Tillé, Y. (2011). A direct bootstrap method for complex sampling designs from a finite population. Journal of the American Statistical Association, 106(494), 534-543. https://doi.org/10.1198/jasa.2011.tm09767

Aquino Guillen, J. (2016). Adaptación a la vida universitaria y resiliencia en becarios. Lima: PUCP.

Banco Mundial. (2014). Panorama general mente, sociedad y conducta. París: Banco Mundial.

Bandura, A. (1989). Human agency in social cognitive theory. American Psychologist, 44(9), $1175-$ 1184. https://doi.org/10.1037/0003-066X.44.9.1175

Becerra, C. y Morales Ballesteros, M. (2015). Validación de la escala de motivación de logro escolar en estudiantes de bachillerato en México. Innovación Educativa, 15(68), 135-152.

Becoña, E. (2006). Resiliencia: Definición, características y utilidad del concepto. Revista de Psicopatología y Psicología Clínica, 11(3), 125-146.

Bernal, C. A. (2012). Metodología de la investigación. Ciudad de México: Pearson.

Cabanach, R. G., Valle, A., Rodríguez, S., Piñeiro, I. y Gonzales, P. (2010). Las creencias motivacionales como factor protector del estrés en estudiantes universitarios. European Journal of Education and Psychology, 3(1), 75-87. https://doi.org/10.30552/ejep.v3i1.49

Cabello, E. y Chirinos, J. L. (2012). Validación y aplicabilidad de encuestas SERVQUAL modificadas para medir la satisfacción de usuarios externos en servicios de salud. Revista Médica Herediana, 23(2), 88-95. https://doi.org/10.20453/rmh.v23i2.1037

Campo Vásquez, R., Granados Ospina, L., Muñoz Ortega, L., Rodríguez Arenas, M. y Trujillo García, S. (2012). Caracterización del avance teórico, investigativo y/o de intervención en resiliencia desde el ámbito de las universidades en Colombia. Universitas Psychologica, 11(2), 545-557.

Campos, Iraurgui, J., Páez, D. y Velasco, C. (2004). Afrontamiento y regulación emocional de hechos estresantes un meta-análisis de 13 estudios. Boletín de Psicología, 82, 25-44.

Canales, A. y De los Ríos, D. (2007). Informe final factores explicativos de la deserción universitaria. Santiago de Chile: Universidad Santiago de Chile.

Cano García, E. y Fernández Ferrer, M. (2015). La visión de los estudiantes de origen extranjero sobre factores y competencias clave para el acceso a la universidad. Pedagogía Social. Revista Interuniversitaria, 26, 263-284. https://doi.org/10.7179/PSRI_2015.26.10

Cassaretto, B. M. y Martínez U. P. (2009). Validación de la escala del sentido del humor en estudiantes universitarios. Revista de Psicología, 27(2), 287-309.

Castilla, H., Caycho, T., Shimabukuro, M. y Valdivia, A. (2014). Percepción del funcionamiento familiar: Análisis psicométrico de la escala APGAR-familiar en adolescentes de Lima. Propósitos y Representaciones, 2(1), 49-78. https://doi.org/10.20511/pyr2014.v2n 1.53 
Collado Agudo, J. (2016, junio). Modelos de ecuaciones estructurales (SEM) para la investigación en contabilidad y auditoría. Conferencia impartida en la I Reunión Internacional de Investigación en Contabilidad y Auditoría (RIICA). Universidad de Cantabria.

Comisión Europea. (2015). Nuevas medidas para impulsar las competencias clave, las capacidades digitales y la dimensión europea de la educación. Bruselas: Comisión Europea.

Coschiza, C. C., Fernández, J. M., Redcozub, G. G., Nievas, M. E. y Ruiz, H. E. (2016). Características socioeconómicas y rendimiento académico. El caso de una universidad argentina. REICE. Revista Iberoamericana sobre Calidad, Eficacia y Cambio en Educación, 14(3), $51-76$.

Crowther, S. y Lau, A. (2019). Migrant polish women overcoming communication challenges in Scottish maternity services: A qualitative descriptive study. Midwifery, 72, 30-38. https://doi.org/10.1016/j.midw.2019.02.004

Cupani, M. (2012). Análisis de ecuaciones estructurales: Conceptos, etapas de desarrollo y un ejemplo de aplicación. Revista Tesis, 2, 186-199.

Cheng, W., Ickes, W. y Verhofstadt, L. (2012). How is family support related to students' GPA scores? A longitudinal study. High Education,64, 399-420.

https://doi.org/10.1007/s 10734-011-9501-4

Datu, J. y Yuen, M. (2018). Predictors and consequences of academic buoyancy: A review of literature with implications for educational psychological. Contemporary School Psychology, 22(3), 207-212. https://doi.org/10.1007/s40688-018-0185-y

Díaz, R. y Arancibia, V. H. (2002). El enfoque de las competencias laborales: Historia, definiciones y generación de un modelo de competencias para las organizaciones y las personas. Psykhe, $11(2), 207-214$.

Escobedo, M. T., Hernández Gómez, J. A., Estebane Ortega, V. y Martínez Moreno, G. (2016). Modelos de ecuaciones estructurales: Características, fases, construcción, aplicación y resultados. Ciencia Ë Trabajo, 55, 16-22. https://doi.org/10.4067/So7 18-24492016000100004

Farnos Miró, J. (2013). Paradigmas en la investigación educativa. Recuperado de https://juandomingofarnos.wordpress.com/2010/04/07/

Farnos Miró, J. (2010). Aprendizaje permanente y disruptivo, los nuevos ejes de la sociedad. Recuperado de https://juandomingofarnos.wordpress.com/2013/05/18/

Frías-Navarro, D. y Pascual Soler, M. (2012). Prácticas del análisis factorial exploratorio en la investigación sobre conducta del consumidor y marketing. Suma Psicológica, 19(1), 47-58.

Gan, Y. y Shang, J. (2007). Coping flexibility and locus of control as predictors of burnout among Chinese college students. Social Behavior and Personality, 35(8), 1087-1098.

https://doi.org/10.2224/sbp.2007.35.8.1087

Garbanzo Vargas, G. M. (2013). Factores asociados al rendimiento académico en estudiantes universitarios desde el nivel socioeconómico: Un estudio en la Universidad de Costa Rica. Revista Electrónica Educare, 17(3), 57-87.

Gargallo López, B., Garfella Esteban, P. R., Sánchez Peris, F., Ros, R. C. y Serra, C. B. (2009). La influencia del autoconcepto en el rendimiento académico en estudiantes universitarios. Revista Española de Orientación y Psicopedagogía, 20(1), 16-28.

Gaxiola, J., González Lugo, S., Contreras Hernández, Z. y Gaxiola Villa, E. (2012). Predictores del rendimiento académico en adolescentes con disposiciones resilientes y no resilientes. Revista de Psicología, 30(1), 47-74. 
Gayles, J. (2005). Playing the game and paying the price: Academic resilience among three highachieving African American males. Anthropology and Education Quarterly, 36(3), 250-264. https://doi.org/10.1525/aeq.2005.36.3.250

Giannuzzo, A. N. (2010). Los estudios sobre el ambiente y la ciencia ambiental. Scientle \& Studia, 8(1), 129-56. https://doi.org/10.1590/S1678-31662010000100006

Girardo, C., Martínez Pellegrini, S., Salgado Locela, L. H. y Costamagna, P. (2016). Desarrollo de capacidades individuales y colectivas en estudios de posgrado y su incidencia en los territorios. Territorios, 34, 215-236. https://doi.org/10.12804/territ34.2016.09

Gómez-Collado, M. E. (2012). La percepción de los estudiantes sobre el programa de tutoría académica. Convergencia. Revista de Ciencias Sociales, 58, 209-233.

González, M. y Landero, R. (2007). Cuestionario de afrontamiento del estrés: Validación en una muestra mexicana. Revista de Psicología Clínica, 12(2), 189-198.

https://doi.org/10.5944/rppc.vol.12.num.3.2007.4044

Guardia Olmos, J. (2016). Esquema y recomendaciones para el uso de los modelos de ecuaciones estructurales. Revista de Estudios e Investigación en Psicología y Educación, 3(2), 75-80. https://doi.org/10.17979/reipe.2016.3.2.1847

Guzmán Brito, M. P. (2012). Modelo predictivo y explicativo del rendimiento académico universitario. Caso de una institución privada en México. Madrid: Universidad Complutense de Madrid.

Hernández, E. y Rovira-Millán, L. V. (2016). Revisión de las propiedades psicométricas y estructura interna de la escala de compromiso organizacional usando el modelo de ecuaciones estructurales. Revista Puertorriqueña de Psicología, 27(1), 166-182.

Kennett, D. J. y Keefer, K. (2006). Impacto de los recursos aprendidos y las teorías de la inteligencia en el logro académico de los estudiantes universitarios. Psicología de la Educación, 26(3), 441457.

Knezevic, M. y Knezevic, J. (2016). The development of self-structures and active coping. interdisciplinary description of complex systems. Interdisciplinary Description of Complex Systems, 14(2), 246-252. https://doi.org/10.7906/indecs.14.2.13

Kormos, J. (2012). The role of individual differences in L2 writing. Journal of Second Language Writing, 21(4), 390-403. https://doi.org/10.1016/j.jslw.2012.09.003

Kötter, T., Tautphäus, Y., Scherer, M. y Voltmer, E. (2014). Health-promoting factors in medical students and students of science, technology, engineering, and mathematics: Design and baseline results of a comparative longitudinal study. BMC Medical Education, 14(134), 1-13. https://doi.org/10.1186/1472-6920-14-134

Kuo, B. (2014). Coping, acculturation and psychological adaptation among migrants: A theoretical and empirical review and synthesis of the literature. Health Psychology E Behavioural Medicine, 2(1), 16-33. https://doi.org/10.1080/2 1642850.2013.843459

Lamas, H. A. (2015). Sobre el rendimiento escolar. Propósitos y Representaciones, 3(1), 313-386.

Lievens, F. y Sackett, P. (2011). La validez de la evaluación de habilidades interpersonales a través de pruebas situacionales de juicio para predecir el éxito académico y el desempeño laboral. Journal of Applied Psychology, 96(5), 927-940. https://doi.org/10.1037/a0023496

Limonero, J. T., Mateo, D., Maté-Mendez, J., González-Barboteo, J., Bayés, R., Bernaus, M., y Viel, S. (2012). Evaluación de las propiedades psicométricas del cuestionario de detección de malestar emocional en pacientes oncológicos. Gaceta Sanitaria, 26(2), 145-152. https://doi.org/10.1016/j.gaceta.2011.07.016 
Limonero, J., Tomás-Sábado, J., Fernández-Castro, J., Gómez-Romero, J. y Ardilla-Herrero, A. (2012). Estrategias de afrontamiento resilientes y regulación emocional: Predictores de satisfacción con la vida. Behavioral Psychology, 20(1), 183-196.

Lopéz-Cortón, A. (2015). Factores predictores de procesos resilientes en jóvenes universitarios. A Coruña: Departamento de Psicología.

López-Vargas, O., Hederich-Martínez, C. y Camargo-Uribe, A. (2011). Estilo cognitivo y logro académico. Educ.Educ, 14(1), 67-82. https://doi.org/10.5294/edu.2011.14.1.4

Lugo-Márquez, C. I., Guerrero-Mojica, N., Castañeda-Guerrero, M., Gámez-Roque, N. F., Martínez-García, I. y Padilla-Muñoz, J. (2016). Resiliencia y factores de riesgo en estudiantes universitarios al inicio de su formación profesional. Revista de Enfermería del Instituto Mexicano del Seguro Social, 24(3), 171-6.

Luthar, S. S., Cicchetti, D. y Becker, B. (2000). La construcción de la resiliencia: Una evaluación crítica y directrices para el trabajo futuro. Child Development, 71(3), 543-562. https://doi.org/10.1111/1467-8624.00164

Mancini, A. D. y Bonanno, G. A. (2009). Predictors and parameters of resilience to loss: Toward and individual differences model. Journal of Personality, 77(6), 1805-1832. https://doi.org/10.1111/j.1467-6494.2009.00601.x

Martin, A. J. y Marsh, H. W. (2009). Academic resilience and academic buoyancy: Multidimensional and hierarchical conceptual framing of causes, correlates and cognate constructs. Oxford Review of Education, 35(3), 353-370. https://doi.org/10.1016/j.lindif.2013.06.006

Martin, A. J., Ginns, P., Brackett, M., Malmberg, L. E. y Hall, J. (2013). Academic buoyancy and psychological risk: Exploring reciprocal relationships. Learning and Individual Differences, 27, 128-133. https://doi.org/10.1080/03054980902934639

Medrano, L. (2010). Autoeficacia académica y su relación con las emociones positivas y negativas en ingresantes universitarios. Recuperado de https://www.aacademica.org/o00-031/450

Medrano, L. A. (2011). Modelo social cognitivo del rendimiento académico en ingresantes universitarios. La contribución de la autoeficacia social académica. Revista Tesis, 1, 87-106.

Medrano, L., Liporace, M. y Pérez, E. (2014). Sistema de evaluación computarizado de satisfacción académica para estudiantes universitarios de primer año. Revista Electrónica de Investigación en Psicología de la Educación, 12(2), 541-562.

Minulescu, M. (2015). Is university capable to build resilience in students? Procedia. Social and Behavioral Sciences, 180, 1628-1631. https://doi.org/10.1016/j.sbspro.2015.02.317

Monacis, L., Sinatra, M., Tanucci, G., Taurino, A. y De Palo, V. (2014). Hacia una comprensión de los factores predictivos y protectores en el juego. Procedia Social and Behavioral Sciencies, 114, 622-627. https://doi.org/10.1016/j.sbspro.2013.12.757

Muñoz Garrido, V. y De Pedro Sotelo, F. (2004). Educar para la resiliencia: Un camino de mirada en situaciones de riesgo social. Revista Complutense de Educación, 16(1), 107-124.

OCDE. (2013). Panorama de la educación. Madrid: Santillana. https://doi.org/10.1787/eag-2012es

Olivas, A. y Barraza, A. (2016). Expectativas de autoeficacia académica en alumnos de un bachillerato técnico de la Ciudad de Durango. Ciudad de México: Universidad Pedagógica de Durango.

Palomar, J. y Gómez Valdez, N. (2010). Desarrollo de una escala de medición de la resiliencia con mexicanos. Interdisciplinaria, 27(1), 7-22. 
Panziera, J. (2014). Estilos de pensamiento y optimismo disposicional en estudiantes de psicología. Mar del Plata: Universidad Nacional de La Plata.

Peralta Díaz, S. C., Ramírez Giraldo, A. F. y Castaño Buitrago, H. (2006). Factores resilientes asociados al rendimiento académico en estudiantes. Psicología desde el Caribe, 17, 196-2 19.

Piemontesi, S. E. y Heredia, D. E. (2009). Afrontamiento ante exámenes: Desarrollos de los principales modelos teóricos para su definición y medición. Anales de Psicología, 25(1), 102111.

Piemontesi, S. E., Heredia, D. E. y Furlan, L. A. (2012). Propiedades psicométricas de la versión en español revisada del inventario alemán de ansiedad ante exámenes (GTAI-AR) en universitarios argentinos. Universitas Psychologica, 11(1), 177-186.

Pizarro Cherre, E. (2017). Relación entre autoestima, resiliencia y rendimiento académico en estudiantes del Centro Preuniversitario de la Universidad Nacional Mayor de San Marcos (CEPREUNMSM). Tesis doctoral. Universidad Nacional Mayor de San Marcos, Perú.

Porto Noronha, A. y Alves Lamas, K. (2014). Preditores do comprometimento com a carreira e sua relacão com o desempenho em universitários. Pensamiento Psicológico, 12(2), 65-78.

Prieto Navarro, L., (2006) Aprendizaje activo en el aula universitaria: El caso del projecto basado en problemas. Miscelánea Comillas, 64(124), 173-196.

Rahimi, B., Baetz, M., Bowen, R. y Balbuena, L. (2014). Resilience, stress, and coping among canadian medical students. Canadian Medical Education Journal, 5(1), 5-12

Rebotier, J., López, J. y Pigeon, P. (2013). Las paradojas de la resiliencia: Miradas cruzadas entre Colombia y Francia. Territorios, 28, 127-145.

Rivas, S., Ordoñez, J., Mejía, M. A. y Poveda, J. M. (2017). Resiliencia en estudiantes de medicina de la Universidad de Los Andes. Fermentum, 27(79), 433-442.

Rodríguez Ayán, M. N. y Ruíz Díaz, M. (2011). Indicadores de rendimiento de estudiantes universitarios: Calificaciones versus créditos acumulados. Revista de Educación, 355, 467-492.

Rodríguez, L., Oñate, M. y Mesurado, B. (2017). Revisión del cuestionario de emociones positivas para adolescentes. Propiedades psicométricas de la nueva versión abreviada. Universitas Psychologica, $16(3)$, 1-23. https://doi.org/10.11144/Javeriana.upsy 16-3.rcep

Rovira Collado, J. (2016). Redes sociales en la universidad: Profesionales, académicas y de lectura. Álabe, 13, 33-51. https://doi.org/10.15645/Alabe2016.13.4

Sandín, B. y Chorot, P. (2003). Cuestionario de afrontamiento del estrés (CAE): Desarrollo y validación preliminar. Revista de Psicopatología y Psicología Clínica, 8(1), 39-54. https://doi.org/10.5944/rppc.vol.8.num.1.2003.3941

Sanz Oro, R. (2010). El profesor como tutor: Un reto a consolidar en el ejercicio profesional de la orientación. REOP: Revista Española de Orientación y Psicopedagogía, 21(2), 346-357. https://doi.org/10.5944/reop.vol.21.num.2.2010.11537

Sineace. (2016). Modelo de acreditación para programas de estudios de educación superior universitaria. Recuperado de www.sineace.gob.pe

So-Hee, L. y Sang-Sook, H. (2016). A predictive model on north Korean refugees' adaptation to south Korean society: Resilience in response to psychological trauma. Asian Nursing Research, 10, 164-172. https://doi.org/10.1016/j.anr.2016.04.003

Sola Martínez, T., Aznar Díaz, I., Romero Rodríguez, J. y Rodríguez-García, A. M. (2019). Eficacia del método flipped clasroom en la universidad: Metaanálisis de la producción científica de impacto. REICE. Revista Iberoamericana sobre Calidad, Eficacia y Cambio en la Educación, $17(1$,, 25-38. https://doi.org/10.15366/reice2019.17.1.002 
Stocker, J. y Faría, L. (2012). Competência percibida no ensino secundário: Do conceito à avaliacao através de um questionário compósito. Psicología. Revista da Associacao Portuguesa Psicologia, 26(2), 113-140. https://doi.org/10.17575/rpsicol.v26i2.273

Sucre, F. y Garrett, M. B. (2016). Educación basada en competencias en línea: Lecciones de EEUU para América Latina. The dialogue leadership for the Americans. Recuperado de http://repositorio.minedu.gob.pe/handle/123456789/4845

Tadayon, R. (2011-2012). Bandura's social learning theory $\&^{\circ}$ social cognitive learning theory. Recuperado de www.simplypsychology.org/bandura.html

Tomás, J. M., Sancho, P., Melendez, J. C. y Mayordomo, T. (2012). Resilience and coping as predictors of general well-being in the ederly: A structural equation modeling approach. Aging \& Mental Health, 16(3), 317-326. https://doi.org/10.1080/13607863.2011.615737

Torrecillas, A., Rodríguez-Mateo, H., Díaz-Negrín, M. E. y Luján Henríquez, I. (2016). Valor predictivo de los moldes de la mente sobre los factores de personalidad. International Journal of Developmental and Educational Psychology 2(1), 295-306. https://doi.org/10.17060/ijodaep.2016.n1.v2.301

Tristán López, A. y Molgado Ramos, D. (2007). Tablas de validez de contenido. San Luis Potosí: Instituto de Evaluación e Ingeniería Avanzada.

Uriarte, J. (2006). Construir la resiliencia en la escuela. Revista de Psicodidáctica, 11(1), 7-24.

Villalta Paúcar, M. (2010). Factores de resiliencia asociados al rendimiento académico en estudiantes de contextos de alta vulnerabilidad social. Revista de Pedagogía, 31(88), 159-188.

Vinod, H. D. (2010). Advances in social science research using R. Londres: Springer. https://doi.org/10.1007/978-1-4419-1764-5

Weier, M. y Lee, C. (2015). How do Australian university students understand adulthood? Australian Psychologist, 50, 157-167. https://doi.org/10.1111/ap.12091

Yamamoto, T. (2017). Introduction to casual mediation analysis using $R$. Recuperado de http://ww2.amstat.org/misc/webinarfiles/MHS3-09-2017.

Yan Lee, T., Kiu Cheung, C. y Man Kwong, W. (2012). Resilience as a positive youth development construct: A conceptual review. The Scientific World Journal, 2012. https://doi.org/10.1100/2012/390450

Zimmerman, B. J., Bandura, A. y Martínez-Pons, M. (1992). Self-motivation for academic attainment: The role of self-efficacy beliefs and personal goal setting. American Educational Research Journal, 29(3), 663-676. https://doi.org/10.3102/00028312029003663

\section{Breve CV del autor}

\section{Orlando Tipismana}

Psicólogo clínico, especialista en modificación de conducta y terapia del comportamiento. Especialista en gestión de la calidad de Instituciones Educativas y Especialista en autoevaluación y acreditación universitaria ambas en la PUCP. Posee formación en Calidad Educativa Universitaria en UNMSM. Especialista en Entornos Virtuales de Aprendizaje en la CAEU-OIE. Diplomado en especialización en Didáctica Universitaria y en Herramientas e Investigación Universitaria (Universidad Norbert Winner). Egresado de Maestría en Psicología y Clínica y de la Salud (Universidad Nacional Mayor de San Marcos). Egresado de Maestría en Educación Superior, con Mención en Docencia e Investigación Universitaria en la Universidad Científica del Sur. Actualmente es Docente 
en la Facultad de Ciencias de la Salud. Especialista en Políticas Públicas en Salud Mental. Capacitador en Gestión de la Calidad Hospitalaria. ORCID ID: https://orcid.org/ooooo002-6548-8890. Email: orlandotipismana@gmail.com 